\title{
Concept of Field Modes and the Behavior of the Magnetohydrodynamic Field
}

\author{
Francts H. Claugser \\ The Johns Hopkins University, Baltimore, Maryland \\ (Received 3 July 1962; revised manuscript received 14 November 1962)
}

\begin{abstract}
A method for studying the behavior of fields by splitting their behavior into independent field modes is presented. The method is used to explore the characteristics of steady, two-dimensional, linearized magnetohydrodynamic fields with finite viscosity and resistivity and arbitrary orientation of the magnetic vector relative to the velocity vector.

It is shown that in general boundary layers and wakes cease to exist in magnetohydrodynamics. Their place is taken by diffusing waves which, in reality, are the fields of a set of viscous-resistive sources, vortices, poles and currents whose field lines are strongly oriented along the characteristic wave directions. When the viscosity and resistivity are equal, these waves diffuse in a simple and independent way, but when these quantities are not equal, the diffusing waves generate a new kind of wake which is located, veil-like, in the fan-shaped region between the two wave directions. These wakes are fed from the differential diffusion of the primary waves. In the special case for which the resistivity is much greater than the viscosity, a new type of pseudo boundary layer is shown to exist in the velocity field. When the viscosity is much greater than the resistivity, this pseudo boundary layer occurs in the magnetic field.
\end{abstract}

\section{INTRODUCTION}

$\mathrm{T}$ HE purpose of the present article is twofold. First we present a method for exploring the magnetohydrodynamic field in which the behavior of the field is split into independent modes of behavior which, when used together, represent the most general behavior that is possible for the field. Second, we use this method to explore steady, twodimensional linearized fields with finite viscosity and resistivity and with constant properties. We shall not assume that the magnetic and velocity fields are aligned.

Previous investigations ${ }^{1-3}$ have revealed that the

${ }^{1}$ H. Alfvén, Cosmical Electrodynamics (Oxford University Press, New York, 1950).

2 H. Hasimoto, Revs. Modern Phys. 32, 860 (1960).

3 T. G. Cowling, Magnetohydrodynamics (Interscience Publishers, London, 1957); D. M. Dix, Aerospace Corp. Rept. No. TDR-930(2230-03) TR-2 (1962); M. B. Glauert, J. Fluid Mechanics 10,276 (1961); F. A. Goldsworthy, ibid. 11, 519 (1961); M. C. Gourdine, Jet Propulsion Laboratory, Tech. Rept. 32-3 (1960); H. Grad, Revs. Modern Phys. 32, 4 (1960); H. P. Greenspan, J. Fluid Mechanics 9, 455 (1960); Quart. Appl. Math. 18, 4 (1961); H. Hasimoto, Phys. Fluids 2, 5 (1959); 2, 3 (1959); R. H. Levy, Avco Research Rept. 124 (1961); W. S. Lewellen, Air Force Office of Scientific Research TN-59-927 (1959); S. Lundquist, Arkiv Fysik 5, 297 (1952); P. S. Lykoudis, Proceedings of the Twelfth International Astronautical Congress (Springer-Verlag, Berlin, 1959), p. 168; J. E. McCune, J. Fluid Mechanics 7, 449 (1960); J. E. McCune and E. L. Resler, Jr., J. Aero/Space Sci. 27, 493 (1960); E. L. Resler, Jr., Revs. Modern Phys. 32, 866 (1960); E. L. Resier, Jr., and J. E. McCune, The Magnetohydrodynamics of Conducting Fluids, edited by D. Bershader (Stanford University Press, Stanford, California, 1959), p. 120; Revs. Modern Phys. 32, 848 (1960); E. L. Resler, Jr., and W. R. Sears, J. Aeronaut. Sci. 25, 235 (1959); Z. Angew. Math. Phys. 9b, Fasc. 5/6, 509 (1958); L. E. Ring, J. Fluid Mechanics 11, 417 (1961); W. R. Sears, J. Aero/Space Sei. 28, 249 (1961); ARS J. 29, 397 (1959); Revs. Modern Phys. 32, 701 (1960); Astronaut. Acta 7, Fasc. 2-3, 223 (1961); W. R. Sears and E. L. Resler, Jr., J. Fluid Mechanics 5, 257 (1959); Advances magnetohydrodynamic field can behave in a fascinating variety of ways. It has become a challenging task to understand the full import of the many new phenomena that have appeared. It has seemed worthwhile to develop a method for exploring these phenomena which would permit their complex patterns of behavior to be split into simpler patterns in much the same way that the behavior of linear electrical and mechanical systems can be split into a set of simpler modes. Such a procedure is quite common for systems described by ordinary differential equations, but it has been used little for systems that are described by partial differential equations. ${ }^{4,5}$

In developing such a method, we have felt it necessary to place upon it a stern set of requirements. Not only must it present the behavior of the field described by the partial differential equations as a finite set of simpler modes of behavior, but in the process it must not be permitted to lose or overlook any mode of behavior. Thus, we not only demand that it present a simplified picture of the behavior of the field but we also demand that the picture so presented be completely representative of all the characteristics of the system.

in Aeronaut. Sci. 4, 657 (1961); A. R. Seebass, Quart. Appl. Math. 19, 231 (1961); K. Stewartson, J. Fluid Mechanics 8, 82 (1962); Proc. Cambridge Phil. Soc. 52, 301 (1956); Ko Tamada, Air Force Office of Scientific Research 1087 (1961); 1551 (1961).

${ }_{4}^{4}$ L. S. G. Kovasznay, J. Aeronaut. Sci. 20, 657 (1953); L. S. G. Kovasznay and B. T. Chu, J. Fluid Mechanics 3, 494 (1958).

5 P. A. Lagerstrom, High Speed Aerodynamics and Jet Propulsion (Princeton University Press, Princeton, New Jersey), Vol. 4. 
We shall see that for the magnetohydrodynamic field we have chosen to investigate, the complete behavior of the field can be split into six independent field modes. Each mode is shown to possess a fundamental solution ${ }^{6}$ which has a distinctive but relatively simple field pattern associated with it. These fundamental solutions, when distributed throughout the plane, represent the most general fields that are possible for the system. The fundamental fields are studied in some detail and it is shown that they reveal a number of new characteristics of the magnetohydrodynamic field.

\section{EQUATIONS OF THE MAGNETOHYDRODYNAMIC FIELD}

For the electromagnetic field, we have Faraday's law $\partial \mathbf{B} / \partial t+\nabla \times \mathbf{E}=\mathbf{0}$, Ampere's law $\nabla \times \mathbf{H}-$ $\partial \mathbf{D} / \partial t-\mathbf{j}=0$, the divergence conditions $\nabla \cdot \mathbf{B}=\mathbf{0}$, $\nabla \cdot \mathbf{D}=\sigma$, and Ohm's law $\mathbf{E}+\mathbf{V} \times \mathbf{B}=\kappa \mathbf{j}$. Here, $\kappa$ is the resistivity of the medium and the other quantities have their conventional meaning.

The equations for the fluid flow field are the mass conservation law $\partial \rho / \partial t+\nabla \cdot(\rho \mathbf{V})=0$ and the momentum conservation law $\nabla \cdot \mathrm{T}=(\partial / \partial t)(\rho \mathrm{V})$, where the stress tensor $T$ is composed of the following:

Electromagnetic stress:

Inertial stress: $-\rho \mathrm{VV}$

$$
\mathrm{BH}+\mathrm{ED}-\mathrm{I}\left(\frac{1}{2} \mathrm{~B} \cdot \mathrm{H}+\frac{1}{2} \mathrm{E} \cdot \mathrm{D}\right)
$$

Pressure stress: $-p \mathrm{I}$,

Viscous stress: $\rho \nu$ def V.

In these expressions, $\mathbf{I}$ is the unit tensor, $\nu$ is the kinematic viscosity, def is the deformation operator, and the remainder of the symbols have their usual meaning.

In the present paper we restrict our attention to steady, two-dimensional, incompressible flows that are electrically neutral and in which the fluid has constant viscosity and resistivity as well as constant dielectric and magnetic constants $\epsilon$ and $\mu$. We assume there is both a dominant velocity and a dominant magnetic field around which we may linearize our equations. In a set of axes moving with the undisturbed flow, the gross electric field is zero. The perturbations in current and electric field are perpendicular to the plane of the two-dimensional flow.

It should be emphasized that we do not assume that the velocity and the magnetic field are aligned, as is so often done. We shall soon see that the

- H. Bateman, Partial Differential Equations of Mathematical Physics (Cambridge University Press, Cambridge, 1932). aligned fields case is a very special case. In it, many of the general magnetohydrodynamic concepts are lost. The nonaligned fields case is a bit more difficult to study, but the greater breadth of the concepts that are uncovered more than compensates for the additional difficulties. For simplicity, we agree to measure the variables, $B=\mu H$ and $E=\epsilon D$, in units of (Alfvén) velocity. Thus, we replace $(\mu / \rho)^{\frac{1}{2}} H$ and $B /(\mu \rho)^{\frac{1}{2}}$ simply by $H$ and $(\epsilon / \rho)^{\frac{1}{3}} E$ and $D /(\rho \epsilon)^{\frac{1}{2}}$ simply by $E$ where the new $H$ and new $E$ are measured in velocity units. We also replace

$$
p / \rho+\mathrm{B} \cdot \mathrm{H} / 2 \rho+\mathrm{E} \cdot \mathrm{D} / 2 \rho
$$

by $P$. Next we linearize the above set of equations, eliminating $\mathbf{j}$ and $\mathbf{E}$ and putting $\mathbf{V}=(U+u, v)$ and $\mathrm{H}=\left(H_{x}+h_{x}, H_{y}+h_{y}\right)$. Once having made the linearization, we do not make any further approximation. We shall proceed just as though there exists, somewhere, a fluid that obeys exactly the linearized equations.

When the above steps have been taken, we find that our magnetohydrodynamic field is described by five variables, $u, v, h_{x}, h_{y}$, and $P$, which are governed by the five equations

$$
\begin{gathered}
U \frac{\partial u}{\partial x}+\frac{\partial P}{\partial x}-\nu \nabla^{2} u-H_{x} \frac{\partial h_{x}}{\partial x}-H_{y} \frac{\partial h_{x}}{\partial y}=0 \\
U \frac{\partial v}{\partial x}+\frac{\partial P}{\partial y}-\nu \nabla^{2} v-H_{x} \frac{\partial h_{y}}{\partial x}-H_{y} \frac{\partial h_{y}}{\partial y}=0 \\
\kappa\left(\frac{\partial h_{y}}{\partial x}-\frac{\partial h_{x}}{\partial y}\right)-H_{y} u+H_{x} v-U h_{y}=0 \\
\frac{\partial u}{\partial x}+\frac{\partial v}{\partial y}=0 \\
\frac{\partial h_{x}}{\partial x}+\frac{\partial h_{y}}{\partial y}=0 .
\end{gathered}
$$

It should be pointed out that some authors replace the third of the above equations by the pair of equations

$$
\begin{aligned}
& U \frac{\partial h_{x}}{\partial x}-H_{x} \frac{\partial u}{\partial x}-H_{y} \frac{\partial u}{\partial y}-\kappa \nabla^{2} h_{x}=0 \\
& U \frac{\partial h_{y}}{\partial x}-H_{x} \frac{\partial v}{\partial x}-H_{y} \frac{\partial v}{\partial y}-\kappa^{2} \nabla^{2} h_{y}=0
\end{aligned}
$$

and omit the equation

$$
\partial h_{x} / \partial x+\partial h_{y} / \partial y=0
$$

as being superfluous. While this procedure is correct, it is accomplished by applying operators which raise the order of the equations and introduce spurious 
modes of behavior which do not check the original physical laws. In the present paper we have been careful to avoid such steps because we want to obtain all of the legitimate modes of behavior and only those.

\section{FACTORIZATION OF THE SYSTEM OPERATOR: THE CONCEPT OF BEHAVIORAL MODES}

We now set out to explore the behavior of the fields governed by the above equations. Suppose we were to select any one of the five field variables and eliminate the others. Regardless of which variable we select the result will always be of the same form: a fixed operator, acting upon the selected variable, equated to zero. This operator, which characterizes our field, is formed from the determinant of the partial differential operators appearing in the above equations, viz:

$$
\left|\begin{array}{ccccc}
U \frac{\partial}{\partial x}-\nu \nabla^{2} & 0 & -H_{x} \frac{\partial}{\partial x}-H_{y} \frac{\partial}{\partial y} & 0 & \frac{\partial}{\partial x} \\
0 & U \frac{\partial}{\partial x}-\nu \nabla^{2} & 0 & -H_{x} \frac{\partial}{\partial x}-H_{y} \frac{\partial}{\partial y} \frac{\partial}{\partial y} \\
-H_{y} & H_{x} & -\kappa \frac{\partial}{\partial y} & \kappa \frac{\partial}{\partial x}-U & 0 \\
\frac{\partial}{\partial x} & \frac{\partial}{\partial y} & 0 & 0 & 0 \\
0 & 0 & \frac{\partial}{\partial x} & \frac{\partial}{\partial y} & 0
\end{array}\right|
$$

When expanded this system operator becomes

$$
\begin{aligned}
\nabla^{2}\left[( U \frac { \partial } { \partial x } - \nu \nabla ^ { 2 } ) \left(U \frac{\partial}{\partial x}-\right.\right. & \left.\kappa \nabla^{2}\right) \\
& \left.-\left(H_{x} \frac{\partial}{\partial x}+H_{u} \frac{\partial}{\partial y}\right)^{2}\right],
\end{aligned}
$$

so that for any variable we have

$$
\begin{aligned}
\nabla^{2}\left[\left(U \frac{\partial}{\partial x}\right.\right. & \left.-\nu \nabla^{2}\right)\left(U \frac{\partial}{\partial x}-k \nabla^{2}\right) \\
& \left.-\left(H_{x} \frac{\partial}{\partial x}+H_{y} \frac{\partial}{\partial y}\right)^{2}\right]\left(u, v, h_{x} h_{y}, P\right)=0 .
\end{aligned}
$$

We notice that the system operator consists of two factors: one is the Laplace operator and the other is a more complex operator containing both the viscosity and the resistivity.

At this point, we take a decisive step and utilize the linear technique used so commonly in vibration theory, quantum mechanics, etc., namely; when the system operator can be split into commuting factors, then correspondingly the behavior of the system can be split into the sum of independent modes of behavior. Each mode is associated with and governed by one of the factors thus split off. Symbolically, if $\mathcal{L}$ is the system operator and $\xi$ is a typical system variable obeying the equation $\mathscr{L} \xi=0$, and if $\mathscr{L}$ factorizes into $\mathscr{L}_{1}$ and $\mathfrak{L}_{2}$, i.e., $\mathfrak{L} \equiv \equiv \mathfrak{L}_{1} \mathfrak{L}_{2}$, then $\xi$ splits into two independent modes, $\xi_{1}$ and $\xi_{2}$, such that $\xi=\xi_{1}+\xi_{2}$, where the modes are separately governed by $\mathfrak{L}_{1} \xi_{1}=0$ and $\mathfrak{L}_{2} \xi_{2}=0$.

This splitting into independent modes of behavior is doubly significant. Instead of being forced to study the behavior of a system as a complicated whole, we may split the behavior into modes and study the modes separately. (Note we are not splitting the system into parts-just its behavior patterns.) Furthermore, we lose nothing in the process because, in linear systems, we can ensure that all patterns of behavior appear in one or the other of the modes and none slip through our net.

Such a splitting into modes is not a common procedure for partial differential equations. As we shall see presently, it is a most powerful device for exploring the basic behavior of unknown fields.

\section{GENERAL DISCUSSION OF MODES BASED ON THE FACTORS OF THE SYSTEM OPERATOR}

Before we seek detailed expressions for the modes which occur in magnetohydrodynamics, it is instructive to discuss those properties of the modes which can be inferred directly from the system operator. The easiest way to do this is to go back to the modes of the basic fields involved and to trace their development as they are brought together and allowed to interact. As a framework for such a study, there is given in Table I the full 
TABLE I. Matrix of operator coefficients for the magnetohydrodynamic field and for various elementary fields.

\begin{tabular}{|c|c|c|c|c|c|c|}
\hline \multicolumn{7}{|c|}{ VARIABLES } \\
\hline$u$ & y & $\mathbf{P}$ & $h_{x}$ & hy & $\mathrm{i}$ & EQUATIONS \\
\hline 0 & 0 & 0 & $\frac{\partial}{\partial y}$ & $-\frac{\partial}{\partial x}$ & 1 & AMPERE'S LAW \\
\hline$c$ & 0 & 0 & $\frac{\partial}{\partial x}$ & $\frac{\partial}{\partial y}$ & 0 & $\begin{array}{l}\text { MAGNETIC } \\
\text { DIVERGENCE }\end{array}$ \\
\hline$U \frac{\partial}{\partial x}$ & $\begin{array}{l}0 \\
\nu \nabla^{2}\end{array}$ & $\begin{array}{l}\frac{\partial}{\partial x} \\
\frac{\partial}{\partial y}\end{array}$ & $\begin{array}{c}-H x \frac{\partial}{\partial x}-H_{y} \frac{\partial}{\partial} \\
0\end{array}$ & $\begin{array}{c}0 \\
-H_{x} \frac{\partial}{\partial x}-H_{y} \frac{\partial}{\partial y}\end{array}$ & $\begin{array}{l}0 \\
0\end{array}$ & $\left\{\begin{array}{l}\text { STRESS } \\
\text { AND } \\
\text { MOMENTUM }\end{array}\right.$ \\
\hline$\frac{\partial}{\partial x}$ & $\frac{\partial}{\partial y}$ & 0 & 0 & 0 & 10 & $\begin{array}{l}\text { MASS } \\
\text { CONSERVATION }\end{array}$ \\
\hline$-H_{y}$ & $H_{x}$ & 0 & 0 & $-U$ & $k$ & OHM'S LAW \\
\hline
\end{tabular}

I Fluid flow field.

II Magnetic field in free space.

III Magnetic field in stationary, perfectly conducting fluid.

IV Magnetohydrodynamic field of perfectly conducting fluid.

$\mathrm{V}$ Magnetohydrodynamic field of viscous resistive fluid.

matrix of operator-coefficients for the magnetohydrodynamic field. (For clarity, we have used the original field equations with the current appearing as one of the field variables.) We shall single out from this table the equations for each of the basic fields, examine their modes, and trace their development as they are brought together and allowed to interact to form the magnetohydrodynamic modes.

First consider the flow of a perfect fluid. Here the appropriate variables are $u, v$, and $P$, and they are governed by the equations for mass and momentum conservation which appear as group I in Table I (from these equations, the viscosity terms must be deleted). If the determinant of this group is evaluated, the system operator is found to be $U(\partial / \partial x) \nabla^{2}$. As expected, we see here emerging the Laplace operator $\nabla^{2}$. Associated with it are the potential flow modes of perfect fluids. In addition there is a second operator, $U(\partial / \partial x)$, which also represents a full-fledged mode of flow behavior. It corresponds to a large class of inviscid wake and slipstream phenomena. An essential feature of every propulsion device (propellors, rockets, jets, flapping wings, etc.) is a slipstream which trails out behind and whose momentum provides the propulsion thrust. Likewise, all obstacles which experience inviscid drag, such as the Kirchhoff obstacles with sharp edges and separated flows, have inviscid wakes which trail out behind and whose momentum loss accounts for the drag. All of these are examples of the inviscid slipstream or wake mode which is associated with the operator $U(\partial / \partial x)$.

What happens to the above equations when viscosity is added? The system operator becomes $\left[U(\partial / \partial x)-\nu \nabla^{2}\right] \nabla^{2}$. This immediately shows that the potential mode survives unchanged. The wake mode alone is altered, the first-order factor $U(\partial / \partial x)$ becoming the Oseen operator, $U(\partial / \partial x)-\nu \nabla^{2}$. This change causes the inviscid wake to become diffuse as it trails rearwardly. The potential mode is able to describe flows in which the fluid moves along solid walls. But it cannot comply with the conditions of zero sliding velocity at the wall, which characterizes real fluids. In contrast, the viscous mode can produce flows in which the fluid next to the wall is retarded in such a way as to yield zero wall velocity. This retarded fluid moves downstream and as it does so, its momentum loss is transmitted to adjacent fluid by diffusion.

When viscosity is low, the regions of retarded flow shrink until they form boundary layers which envelope all of the solid surfaces. When these layers reach the rear edges of such surfaces, they trail off downstream, forming viscous wakes which gradually diffuse. These concepts, the boundary layer and the viscous wake, are both examples of the viscous mode. They are associated exclusively with the operator $U(\partial / \partial x)-\nu \nabla^{2}$.

Next, let us turn our attention to the magnetic field in free space. Here the current must be zero and the governing equations are $\nabla \cdot h=0$ and $\nabla \times \mathbf{h}=0$, which correspond to group II in Table I. These lead to the system operator $\nabla^{2}$ as would be expected.

Now let us combine the magnetic field with that of a stationary, perfectly conducting fluid. Currents come into play, and since these are unimpeded by resistivity, Ampere's law $\nabla \times \mathbf{h}=\mathbf{j}$ no longer serves as a governing equation for our system. Instead we must look to a balance of forces through the electromagnetic stresses. The appropriate equations for our system are those of group III in Table I. This leads to the system operator $\left[H_{x}(\partial / \partial x)+H_{y}(\partial / \partial y)\right] \nabla^{2}$. Here we see a close parallel with the operator for an inviscid fluid. The Laplace operator appearing here has the same parentage as the Laplace operator of the fluid flow field. Both arise from the force balance laws and neither is related to the Laplace operator of the magnetic field in free space, since the latter traces back to Ampere's law. The operator $H_{x}(\partial / \partial x)+$ $H_{y}(\partial / \partial y)$ is equivalent to the inviscid wake operator $U(\partial / \partial x)$. It leads to an irresistive wave mode having the same properties as the inviscid wake mode, except that the waves are along the magnetic lines rather than along streamlines.

So far we have investigated the fluid-flow field and the magnetic field separately. Now we should 
like to find out what happens when the two are combined. Our first step in this direction is to return to the equations for the flow of an inviscid fluid and simply add the possibility of a magnetic field, but to keep the main field and the resistivity equal to zero. The appropriate equations are those of group IV of Table I with $H_{x}=H_{y}=0$ and with $\nu=0$. The resulting system operator is $[U(\partial / \partial x)]^{2} \nabla^{2}$. Here we have the intriguing result that simply adding the possibility of a magnetic field doubles the inviscid wake mode by doubling the factor $U(\partial / \partial x)$. Where we had one wake before, we now have two, one lying on top of the other.

Next, let us make the magnetic field finite. The system operator becomes

$$
\left[\left(U+H_{x}\right) \frac{\partial}{\partial x}+H_{y} \frac{\partial}{\partial y}\right]\left[\left(U-H_{x}\right) \frac{\partial}{\partial x}-H_{y} \frac{\partial}{\partial y}\right] \nabla^{2} .
$$

A strange transformation has taken place. The two inviscid wake modes have disappeared and their place has been taken by the two Alfvén ${ }^{1}$ wave modes. If we go back and examine the transition carefully, we see that as $H_{x}$ and $H_{y}$ rise from zero, the wakes do not actually disappear, but rather they undergo a metamorphosis and become the Alfven waves. The process is this. Going back to the birth of the magnetic field, the inviscid wake first doubles and then as the magnetic field grows, these two wakes disentangle themselves and turn through the Alfven angles. At this point they become Alfvén waves. Both wakes and individual waves are associated with linear first-order operators. The distinction normally lies in the direction associated with the operator. The magnetic field, by changing the rearward direction of the wakes to diagonal directions, converts them into waves.

This change of the wakes into waves has potentially profound consequences for magnetohydrodynamics. It certainly means that inviscid boundary layers and wakes completely cease to exist in magnetohydrodynamics. All of their rights and privileges are taken over by the Alfvén waves (except for the special case of aligned fields, where there may be either double wakes and boundary layers pointing downstream or one set of wakes and boundary layers pointing downstream and another pointing upstream!).

We now take the final step of considering the full magnetohydrodynamic field with finite viscosity and resistivity. Here we must use the complete set of coefficients of group $\mathrm{V}$ in Table I. This brings us full circle to our original system operator:

$$
\begin{aligned}
\nabla^{2}\left[\left(U \frac{\partial}{\partial x}-\nu \nabla^{2}\right)\left(U \frac{\partial}{\partial x}-\kappa \nabla^{2}\right)\right. & \\
& \left.-\left(H_{x} \frac{\partial}{\partial x}+H_{y} \frac{\partial}{\partial y}\right)^{2}\right]
\end{aligned}
$$

Of the two factors of this operator, the Laplace operator alone has survived unchanged. Its presence indicates that real magnetohydrodynamic fields will have the same purely potential modes that are present in magnetic fields and perfect fluid fields. This is surprising in view of the fact that we are dealing with a fluid having both resistivity and viscosity.

The other factor in the above system operator is the viscous-resistive operator. It is of fourth order and thus is more complex than anything we have encountered so far. In general, it is not possible to split it into simpler factors as long as $\nu$ and $\kappa$ have arbitrary values. Later we shall see that the reason for this is that this operator contains modes of behavior that are not simply the sum of the modes of behavior we have studied so far. Instead, fundamentally new concepts are involved.

Fortunately, however, it is possible to simplify our preliminary investigations. Hasimoto ${ }^{2}$ has shown that when the magnetic Prandtl number is unity, i.e. when $\nu=\kappa$, the viscous-resistive operator does factorize, splitting into the two operators

$$
\left(U+H_{x}\right)(\partial / \partial x)+H_{y}(\partial / \partial y)-\nu \nabla^{2}
$$

and

$$
\left(U-H_{x}\right)(\partial / \partial x)-H_{y}(\partial / \partial y)-\nu \nabla^{2} .
$$

This shows that for this case, the behavior can be split into the sum of two different patterns of behavior, both of which are already familiar to us. To relate the above two operators to our previous work, let us rotate the $(x, y)$ coordinates of the first through the Alf vén angle $\tan ^{-1}\left[H_{y} /\left(U+H_{x}\right)\right]$ and the second through the Alfvén angle $\tan ^{-1}\left[-H_{y} /\left(U-H_{x}\right)\right]$. Let us also introduce the compound velocities $W_{-}=\left[\left(U \pm H_{x}\right)^{2}+H_{y}^{2}\right]^{\frac{1}{2}}$ made up of the fluid velocity $U$ and the magnetic velocities $H_{x}$ and $H_{y}$. With these changes both operators become simply $W_{ \pm}\left(\partial / \partial x^{\prime}\right)-\nu \nabla^{2}$. This is just the Oseen operator ${ }^{5}$ and we immediately see that each of the Alfven waves experiences diffusion in exactly the same way that the wakes and boundary layers of ordinary fluid mechanics do. In the system operator there is not the slightest vestige of the old downstream boundary layer or wake mode of fluid mechanics. We are forced to conclude that for the case $\nu=k$ in real magnetohydrodynamics, as well as in perfect mag- 
netohydrodynamics, the old concepts of boundary layers and wakes cease to exist. No longer will there be retarded layers flowing next to solid surfaces and trailing off downstream. Instead, these functions will be taken over by diffusing Alfvén waves. In these waves, the frictional momentum deficit and the resistive magnetic deficit will be carried outward along the Alfvén waves and diffused there, rather than being carried back over the body itself and trailing off as a wake. To see how this occurs requires more detailed knowledge of these modes than we can obtain from just the factors of the system operator. This detailed knowledge we uncover in the following sections.

We still leave unanswered the intriguing question of what happens to the Alfvén waves when $\nu$ and $\kappa$ are not equal. Clearly, viscosity and resistivity serve to couple them in an inextricable manner that lies outside our usual experience. We shall find that it is not difficult to obtain specific expressions for the modes of the general viscous-resistive operator. However, the exploration of their detailed behavior is not a simple matter. Consequently, our initial efforts in this direction must necessarily be devoted to the case for $\nu=\kappa$. However, after we have achieved an understanding of this case, we shall return to the general case and explore it in detail. Not only shall we find that it presents a fascinating picture of the behavior of the magnetohydrodynamic field, but we shall also find that it shows with great clarity what happens when partial differential operators become unfactorizable.

\section{FORM OF THE FIELD MODES}

Let us now turn to the problem of finding the specific form of each of the field modes. First consider the familiar Laplace operator which is one of the factors of our system operator. This operator, $\partial^{2} / \partial x^{2}+\partial^{2} / \partial y^{2}$ can be factored into two factors, $\partial / \partial x+i \partial / \partial y$ and $\partial / \partial x-i \partial / \partial y$, showing that in reality it represents $t w o$ modes.

First let us look at the mode corresponding to $\partial / \partial x+i \partial / \partial y$. The fact that all five of the field variables $u, v, h_{x}, h_{y}$, and $P$ obey equations of the form $\partial u / \partial x+i \partial u / \partial y=0$ is sufficient to guarantee that the homogeneous field equations can all be satisfied simultaneously. However, we have the additional fact that these equations establish relationships among the field variables. We choose to express this relationship by expressing each of the variables in terms of what we shall call a modal variable. For the mode corresponding to the factor, $\partial / \partial x+i \partial / \partial y$, let us call this modal variable $f$.
It is not difficult to find the relationship of the field variables to this modal variable. They are $u=U f$, $v=i U f, h_{x}=-\left(H_{x}+i H_{y}\right) f, h_{y}=-i\left(H_{x}+i H_{y}\right) f$, and $P=-\left(U^{2}+H_{x}^{2}-H_{y}^{2}+2 i H_{x} H_{y}\right) f$. These relations, along with the equation $\partial f / \partial x+i \partial f / \partial y=0$ completely characterize this field mode.

For the other mode we use the modal variable $g$ which satisfies the equation $\partial g / \partial x-i \partial g / \partial y=0$. This mode has the form $u=U g, v=-i U g$, $h_{x}=-\left(H_{x}-i H_{y}\right) g, h_{y}=i\left(H_{x}-i H_{y}\right) g$, and

$$
P=-\left(U^{2}+H_{x}^{2}-H_{y}^{2}-2 i H_{x} H_{y}\right) g .
$$

The above expressions are complex and it is necessary for us to obtain real expressions for our physical variables. We do this in a way which serves to answer an important question which will confront us later. We pause long enough to lay the groundwork for this question.

It is well known that ordinary differential operators with constant coefficients can always be factored into terms of first order. In constrast, such factorization is usually not possible with partial differential operators. The reader can readily verify that such simple operators as $\partial^{2} / \partial x^{2}-\partial / \partial y$ and $\partial^{2} / \partial x^{2}-$ $\partial^{2} / \partial y^{2}-1$ cannot be factored into first-order operators. This fact is of great significance because it shows that we cannot treat partial differential equations in the same way that we treat ordinary differential equations.

We now ask the question: Can the behavior of unfactorizable operators be split into separate modes and if so, what is the form of such modes? The answer to the first part of this question is yes, as we shall later show repeatedly. To obtain an answer to the second part, we now show that the modes of the factors of the Laplace operator can be expressed as modes of the unfactored operator. The resulting expressions will allow us to deduce the form that the modes of unfactorizable operators will take. A by-product will be the real expressions we are seeking for our potential modes.

The two functions $f$ and $g$ obey the equations $\partial f / \partial x+i \partial f / \partial y=0$ and $\partial g / \partial x-i \partial g / \partial y=0$. Let us introduce two new variables $\phi$ and $\psi$ such that $f=\partial \phi / \partial x-i \partial \phi / \partial y$ and $g=\partial \psi / \partial x+i \partial \psi / \partial y$. An immediate result is that both $\phi$ and $\psi$ satisfy the unfactored operator $\nabla^{2}$, i.e., $\nabla^{2} \phi=0, \nabla^{2} \psi=0$. Furthermore, if we substitute the above expressions for $f$ and $g$ into our previous algebraic modal relationships, we obtain expressions that contain first-order derivatives of $\phi$ and $\psi$. From these results we may infer the following answer to the question posed above. 
Unfactored or unfactorizable operators possess modes such that:

(1) The modal variables of all the modes obey the unfactored operator.

(2) Each modal relationship may contain derivatives up to one less than the order of the unfactored operator.

Returning now to the Laplace operator, we may immediately obtain real expressions for the modes, (since $\phi$ and $\psi$ are real). These are

$$
\begin{array}{rlrl}
u & =U \frac{\partial \phi}{\partial x}, \quad \text { and } & u & =U \frac{\partial \psi}{\partial y}, \\
v & =U \frac{\partial \phi}{\partial y}, & v & =-U \frac{\partial \psi}{\partial x}, \\
h_{x} & =H_{x} \frac{\partial \phi}{\partial x}+H_{y} \frac{\partial \phi}{\partial y}, & h_{x} & =-H_{y} \frac{\partial \psi}{\partial x}+H_{x} \frac{\partial \psi}{\partial y}, \\
h_{y} & =-H_{y} \frac{\partial \phi}{\partial x}+H_{x} \frac{\partial \phi}{\partial y}, & h_{y} & =-H_{x} \frac{\partial \psi}{\partial x}-H_{y} \frac{\partial \psi}{\partial y}, \\
P & =-U u+H_{x} h_{x}+H_{y} h_{y} .
\end{array}
$$

It will be noticed that the form of these modes is the same as that of the usual potential functions and stream functions of complex potential theory. However, here their use is different. $\phi$ and $\psi$ are not real and imaginary parts of the same complex function. Rather they are independent functions and later we shall see how they lead to independent modes of behavior.

Now that we have obtained the Laplacian modes in real form, let us turn to the more difficult problem of finding the modes of the viscous resistive operator, $\left(U \frac{\partial}{\partial x}-\nu \nabla^{2}\right)\left(U \frac{\partial}{\partial x}-\kappa \nabla^{2}\right)-\left(H_{x} \frac{\partial}{\partial x}+H_{y} \frac{\partial}{\partial y}\right)^{2}$.

In general, this operator cannot be factored. It is here that our findings about unfactored operators stand us in good stead. Since we are dealing with a fourth-order operator, we conjecture that we should search for modal relationships that involve up to third-order derivatives of the modal variable. Furthermore, each of the modal variables should satisfy the full fourth-order operator. Such a search is rewarded, after a bit of calculation, by our finding that four independent modal relationships having this form do in fact exist. These constitute a complete set for our fourth-order operator.

If we introduce four modal variables $\lambda, \mu, \chi$, and $\beta$, each of which satisfies an equation of the form

$$
\begin{aligned}
\left(U \frac{\partial}{\partial x}-\nu \nabla^{2}\right)\left(U \frac{\partial}{\partial x}-\kappa \nabla^{2}\right) \lambda & \\
& -\left(H_{x} \frac{\partial}{\partial x}+H_{y} \frac{\partial}{\partial y}\right)^{2} \lambda=0,
\end{aligned}
$$

then we have the following specific forms for the four viscous-resistive modes.

\section{$\lambda$ Mode}

$$
\begin{aligned}
& u=\left(U+H_{x}-\nu \frac{\partial}{\partial x}\right)\left(U \frac{\partial}{\partial x}-\kappa \nabla^{2}\right) \lambda \\
& -\left(U+H_{x}-\kappa \frac{\partial}{\partial x}\right)\left(H_{x} \frac{\partial}{\partial x}+H_{y} \frac{\partial}{\partial y}\right) \lambda, \\
& v=\left(H_{y}-\nu \frac{\partial}{\partial y}\right)\left(U \frac{\partial}{\partial x}-\kappa \nabla^{2}\right) \lambda \\
& -\left(H_{y}-\kappa \frac{\partial}{\partial y}\right)\left(H_{x} \frac{\partial}{\partial x}+H_{y} \frac{\partial}{\partial y}\right) \lambda, \\
& h_{x}=-\left(U+H_{x}-\kappa \frac{\partial}{\partial x}\right)\left(U \frac{\partial}{\partial x}-\nu \nabla^{2}\right) \lambda \\
& +\left(U+H_{x}-\nu \frac{\partial}{\partial x}\right)\left(H_{x} \frac{\partial}{\partial x}+H_{y} \frac{\partial}{\partial y}\right) \lambda, \\
& h_{y}=-\left(H_{y}-\kappa \frac{\partial}{\partial y}\right)\left(U \frac{\partial}{\partial x}-\nu \nabla^{2}\right) \lambda \\
& +\left(H_{y}-\nu \frac{\partial}{\partial y}\right)\left(H_{x} \frac{\partial}{\partial x}+H_{y} \frac{\partial}{\partial y}\right) \lambda,
\end{aligned}
$$

$P=0$.

\section{u Mode}

$$
\begin{aligned}
u & =\frac{\partial}{\partial y}\left(\nu U \frac{\partial}{\partial x}-{ }_{\kappa} H_{x} \frac{\partial}{\partial x}-{ }_{\kappa} H_{y} \frac{\partial}{\partial y}-\nu \kappa \nabla^{2}\right) \mu, \\
v & =-\frac{\partial}{\partial x}\left(\nu U \frac{\partial}{\partial x}-{ }_{\kappa} H_{x} \frac{\partial}{\partial x}-{ }_{\kappa} H_{y} \frac{\partial}{\partial y}-\nu \kappa \nabla^{2}\right) \mu, \\
h_{x} & =-\frac{\partial}{\partial y}\left({ }_{\kappa} U \frac{\partial}{\partial x}-\nu H_{x} \frac{\partial}{\partial x}-\nu H_{y} \frac{\partial}{\partial y}-\nu \kappa \nabla^{2}\right) \mu, \\
h_{y} & =\frac{\partial}{\partial x}\left({ }_{\kappa} U \frac{\partial}{\partial x}-\nu H_{x} \frac{\partial}{\partial x}-\nu H_{y} \frac{\partial}{\partial y}-\nu \kappa \nabla^{2}\right) \mu, \\
P & =0 .
\end{aligned}
$$$$
x \text { Mode }
$$$$
u=\left(U-H_{x}-\nu \frac{\partial}{\partial x}\right)\left(U \frac{\partial}{\partial x}-\kappa \nabla^{2}\right) \chi
$$$$
+\left(U-H_{x}-\kappa \frac{\partial}{\partial x}\right)\left(H_{x} \frac{\partial}{\partial x}+H_{y} \frac{\partial}{\partial y}\right) \chi,
$$$$
v=-\left(H_{y}+\nu \frac{\partial}{\partial y}\right)\left(U \frac{\partial}{\partial x}-\kappa \nabla^{2}\right) \chi
$$$$
-\left(H_{y}+\kappa \frac{\partial}{\partial y}\right)\left(H_{x} \frac{\partial}{\partial x}+H_{y} \frac{\partial}{\partial y}\right) \chi,
$$$$
h_{x}=\left(U-H_{x}-\kappa \frac{\partial}{\partial x}\right)\left(U \frac{\partial}{\partial x}-\nu \nabla^{2}\right) x
$$$$
+\left(U-H_{x}-\nu \frac{\partial}{\partial x}\right)\left(H_{x} \frac{\partial}{\partial x}+H_{y} \frac{\partial}{\partial y}\right) x,
$$ 


$$
\begin{aligned}
h_{y}=-\left(H_{y}\right. & \left.+\kappa \frac{\partial}{\partial y}\right)\left(U \frac{\partial}{\partial x}-\nu \nabla^{2}\right) \chi \\
& -\left(H_{y}+\nu \frac{\partial}{\partial y}\right)\left(H_{x} \frac{\partial}{\partial x}+H_{y} \frac{\partial}{\partial y}\right) \chi,
\end{aligned}
$$

$P=0$.

\section{Mode}

$$
\begin{aligned}
u & =\frac{\partial}{\partial y}\left(\nu U \frac{\partial}{\partial x}+{ }_{\kappa} H_{x} \frac{\partial}{\partial x}+{ }_{\kappa} H_{y} \frac{\partial}{\partial y}-\nu \kappa \nabla^{2}\right) \beta, \\
y & =-\frac{\partial}{\partial x}\left(\nu U \frac{\partial}{\partial x}+{ }_{\kappa} H_{x} \frac{\partial}{\partial x}+{ }_{\kappa} H_{y} \frac{\partial}{\partial y}-\nu \kappa \nabla^{2}\right) \beta, \\
h_{x} & =\frac{\partial}{\partial y}\left({ }_{\kappa} U \frac{\partial}{\partial x}+\nu H_{x} \frac{\partial}{\partial x}+\nu H_{y} \frac{\partial}{\partial y}-\nu \kappa \nabla^{2}\right) \beta, \\
h_{y} & =-\frac{\partial}{\partial x}\left({ }_{\kappa} U \frac{\partial}{\partial x}+\nu H_{x} \frac{\partial}{\partial x}+\nu H_{y} \frac{\partial}{\partial y}-\nu \kappa \nabla^{2}\right) \beta, \\
P & =0 .
\end{aligned}
$$

It would be a formidable task to plunge directly into an investigation of the above expressions. We have seen earlier that this task can be made simpler by first studying the case $\nu=\kappa$ in which the operator splits into two factors. The modes for the factored operator are, of course, not obtained by simply putting $\nu=\kappa$ in the above modal expressions. On the basis of our experience with the Laplace operator, we should expect to be able to remove a common operator-factor from each set of modal relations, reducing each to a simpler first-order expression. The second-order factor so removed should be one of the factors of the fourth-order operator. Consequently, this same maneuver should reduce the order of the equations satisfied by the modal variables from four to two and the resulting operators should be the factors of the original fourth-order operator.

Earlier we found that the factors of the fourthorder operator are

$$
\left(U+H_{x}\right) \frac{\partial}{\partial x}+H_{y} \frac{\partial}{\partial y}-\nu \nabla^{2}
$$

and

$$
\left(U-H_{y}\right) \frac{\partial}{\partial x}-H_{y} \frac{\partial}{\partial y}-\nu \nabla^{2} .
$$

If we examine our four sets of modal relationships, we find that when $\nu=\kappa$, the second of these operators does appear as a common factor throughout both the $\lambda$ and $\mu$ modes, while the first appears throughout the $\chi$ and $\beta$ modes. Consequently, if we introduce four revised modal variables
TABLE II. Form of the viscous resistive modes when $\nu=\kappa$.

\begin{tabular}{ccccc}
\hline & $\lambda^{\prime}$ & $\mu^{\prime}$ & $\chi^{\prime}$ & $\beta^{\prime}$ \\
\hline$u$ & $\left(C+H_{x}\right) \lambda^{\prime}-\nu \frac{\partial \lambda^{\prime}}{\partial x}$ & $\nu \frac{\partial \mu^{\prime}}{\partial y}$ & $\left(U-H_{x}\right) \chi^{\prime}-\nu \frac{\partial \chi^{\prime}}{\partial x}$ & $\nu \frac{\partial \beta^{\prime}}{\partial y}$ \\
$v$ & $H_{y} \lambda^{\prime}-\nu \frac{\partial \lambda^{\prime}}{\partial y}$ & $-\nu \frac{\partial \mu^{\prime}}{\partial x}$ & $-H_{u} \chi^{\prime}-\nu \frac{\partial \chi^{\prime}}{\partial y}$ & $-\nu \frac{\partial \beta^{\prime}}{\partial x}$ \\
$h_{x}$ & $-\left(U+H_{x}\right) \lambda^{\prime}+\nu \frac{\partial \lambda^{\prime}}{\partial x}$ & $-\nu \frac{\partial \mu^{\prime}}{\partial y}$ & $\left(U-H_{x}\right) \chi^{\prime}-\nu \frac{\partial \chi^{\prime}}{\partial x}$ & $\nu \frac{\partial \beta^{\prime}}{\partial y}$ \\
$h_{y}$ & $-H_{y} \lambda^{\prime}+\nu \frac{\partial \lambda^{\prime}}{\partial y}$ & $\nu \frac{\partial \mu^{\prime}}{\partial x}$ & $-H_{y} \chi-\nu \frac{\partial \chi^{\prime}}{\partial y}$ & $-\nu \frac{\partial \beta^{\prime}}{\partial x}$ \\
$P$ & 0 & 0 & 0 & 0 \\
\hline
\end{tabular}

$$
\begin{aligned}
& \lambda^{\prime}=\left(U-H_{x}\right) \frac{\partial \lambda}{\partial x}-H_{y} \frac{\partial \lambda}{\partial y}-\nu \nabla^{2} \lambda, \\
& \mu^{\prime}=\left(U-H_{x}\right) \frac{\partial \mu}{\partial x}-H_{y} \frac{\partial \mu}{\partial y}-\nu \nabla^{2} \mu, \\
& \chi^{\prime}=\left(U+H_{x}\right) \frac{\partial \chi}{\partial x}+H_{x} \frac{\partial \chi}{\partial y}-\nu \nabla^{2} \chi, \\
& \beta^{\prime}=\left(U+H_{x}\right) \frac{\partial \beta}{\partial x}+H_{y} \frac{\partial \beta}{\partial y}-\nu \nabla^{2} \beta,
\end{aligned}
$$

then two essential simplifications occur. First, instead of all four variables obeying a single fourth-order equation, they obey, by pairs, two second-order equations which come from the factors of the fourthorder expression. Thus,

$$
\begin{aligned}
& \left(U+H_{x}\right) \frac{\partial \lambda^{\prime}}{\partial x}+H_{y} \frac{\partial \lambda^{\prime}}{\partial y}-\nu \nabla^{2} \lambda^{\prime}=0, \\
& \left(U+H_{x}\right) \frac{\partial \mu^{\prime}}{\partial x}+H_{y} \frac{\partial \mu^{\prime}}{\partial y}-\nu \nabla^{2} \mu^{\prime}=0, \\
& \left(U-H_{x}\right) \frac{\partial \chi^{\prime}}{\partial x}-H_{y} \frac{\partial \chi^{\prime}}{\partial y}-\nu \nabla^{2} \chi^{\prime}=0, \\
& \left(U-H_{x}\right) \frac{\partial \beta^{\prime}}{\partial x}-H_{y} \frac{\partial \beta^{\prime}}{\partial y}-\nu \nabla^{2} \beta^{\prime}=0 .
\end{aligned}
$$

Second, the modal relationships take on the simple forms given in Table II.

We are now in a position to interpret these findings. Our magnetohydrodynamic field possesses six independent modes of behavior. Two of the modes are potential modes and reflect the familiar behavior of the complex variable theory. The remaining four are viscous-resistive modes and represent an as yet incomprehensible intercoupling of the Alfvén waves with viscous-resistive phenomenon. We have found explicit expressions for all six of the modes and we have explicit equations for all six of the modal variables. For the special case, $\nu=\kappa$, we have found that the Alfven waves cease to be intercoupled by 
viscous-resistive phenomena and instead each wave experiences a diffusion similar to the Oseen diffusion of viscous boundary layers and wakes. In this later case, it is clear that viscous boundary layers and wakes cease to exist and their rights and privileges are taken over by the diffuse Alfvén waves.

\section{CONCEPT OF THE FUNDAMENTAL FIELD MODES}

Now that we have split the behavior of our field into independent modes, our next step is to show that each mode in turn possesses a simple and distinctive pattern of behavior called a fundamental mode and that these fundamental modes may be used to build up the most general patterns of field behavior.

One of the basic concepts of partial differential equations is that of the fundamental solution. ${ }^{6}$ To illustrate what is meant by a fundamental solution, let us suppose that we have an operator $\mathcal{L}$ which acts upon a variable $\xi$ to give another variable $\sigma$, thus $\mathscr{L} \xi=\sigma$. In our case, $\&$ will be one of the modal operators and $\xi$ will be one of the six variables $\phi, \psi, \lambda, \mu, \chi$, and $\beta$. We may always express the operator in the integral form

$$
\begin{aligned}
2 \xi & \equiv \int_{-\infty}^{\infty} \int_{-\infty}^{\infty} \xi\left(x^{\prime}, y^{\prime}\right) K\left(x-x^{\prime}, y-y^{\prime}\right) d x^{\prime} d y^{\prime} \\
& =\sigma(x, y) .
\end{aligned}
$$

Here we have used a kernel having the form $K\left(x-x^{\prime}, y-y^{\prime}\right)$ because this is appropriate for all operators (such as ours) which are unaffected by a translation of coordinates.

If we invert the above relationship in order to solve for $\xi$, we have

$\xi(x, y)=\int_{-\infty}^{\infty} \int_{-\infty}^{\infty} \sigma\left(x^{\prime}, y^{\prime}\right) K^{-1}\left(x-x^{\prime}, y-y^{\prime}\right) d x^{\prime} d y^{\prime}$.

Next, if we take $\sigma$ to be the Dirac function which is zero everywhere except at the origin and there it has a unit integral, then this last relationship becomes

$$
\xi(x, y)=K^{-1}(x, y) .
$$

This shows that the inverse kernel has a very simple interpretation: it is the solution for the field when the "forcing function" on the right-hand side is zero everywhere except at the origin and there it has a unit impulse. We call this the fundamental solution for the operator $\&$.

For operators, such as ours, which are differential operators, the kernels $K$ are highly singular (involving derivatives of Dirac functions) and their inversion by formal methods requires unusual skill. However, when interpreted as we have done above they may frequently be found in elementary ways, as we shall see below.

The significance of the fundamental solution is that it allows us to synthesize the most general patterns of behavior associated with the operator $\&$ from a single specific pattern. And this single specific pattern, i.e., the field of the fundamental solution itself, may be studied once and for all time and thus understood thoroughly.

Let us illustrate the above concepts by the familiar example of the electric field. In conventional notation we have $\nabla^{2} V=\sigma$. Here our operator $\mathscr{L}$ is $\nabla^{2}$. Its formal inversion is difficult. However, when the charge distribution $\sigma$ is zero everywhere except at the origin, and there it has an integral of unity, we say that a unit charge exists at the origin. The solution for this field is $K^{-1}=(1 / 2 \pi) \log r$. This is the fundamental solution we are seeking.

A general solution for the electric field is obtained by placing an arbitrary distribution of charges throughout the plane and summing up their contributions as follows:

$$
\begin{aligned}
V(x, y)=\frac{1}{2 \pi} \int_{-\infty}^{\infty} \int_{-\infty}^{\infty} \sigma\left(x^{\prime}, y^{\prime}\right) \\
\cdot \log \left[\left(x-x^{\prime}\right)^{2}+\left(y-y^{\prime}\right)^{2}\right]^{\frac{1}{2}} d x^{\prime} d y^{\prime} .
\end{aligned}
$$

It will be recognized immediately that this is just a restatement of the steps we have given above, except that here they are couched in the language of electrostatics.

Let us consider a second example. Suppose that we have the first-order equation $a(\partial \xi / \partial x)+b(\partial \xi / \partial y)=\sigma$. In the physical world, such an equation may represent a single family of waves, wakes, or fronts. If $\sigma$ is zero everywhere except for a unit impulse at the origin, it will induce an impulsive wave traveling outward from the origin along the line whose slope is $\tan ^{-1}(b / a)$. (To tell in which direction along the line the wave travels, we must have additional information and this can only be obtained from the physical problem itself.) The fundamental solution is thus a distributed Dirac function which starts at the origin and which lies along the line having a slope $\tan ^{-1}(b / a)$. This function has an integral of $\left(a^{2}+b^{2}\right)^{-\frac{1}{2}}$ per unit length of line.

As a third example let us consider the more common double wave equation

$$
\frac{\partial^{2} \xi}{\partial x^{2}}-\frac{1}{c^{2}} \frac{\partial^{2} \xi}{\partial t^{2}}=\sigma .
$$


It is instructive to build up the fundamental solution from that of the single wave equation. If we put

$$
\eta=\frac{\partial \xi}{\partial x}-\frac{1}{c} \frac{\partial \xi}{\partial t}
$$

then $\eta$ satisfies the single wave equation

$$
\frac{\partial \eta}{\partial x}+\frac{1}{c} \frac{\partial \eta}{\partial t}=\sigma
$$

which as we have just seen, has as fundamental solution a distributed Dirac function extending outward from the origin along the line $x=c t$. This corresponds to a unit impulse at the origin for $\sigma$. Turning now to the equation

$$
\frac{\partial \xi}{\partial x}-\frac{1}{c} \frac{\partial \xi}{\partial t}=\eta
$$

we must obtain a solution for $\xi$ which corresponds not to a single impulse for $\eta$, but to a uniform distribution extending from the origin out along the line $x=c t$. This may be obtained by integration and the resulting fundamental solution for $\xi$ is found to be $(c / 2 \pi) \log [(x-c t)(x+c t)]^{\frac{1}{2}}$. This same result can, of course, be obtained from the solution for Laplace's equation by replacing iy by $c t$.

The above discussion serves to tie in the familiar cases of sources, charges, poles and the like in potential theory and wave theory with more general examples of fundamental solutions. Clearly, fundamental solutions are of immense value in exploring the behavior of fields. In the same way that the behavior of an entire field is the superposition of the behavior of its individual modes, so the behavior of a given mode is the superposition of the behavior of its fundamental solutions.

\section{FUNDAMENTAL MODES OF MAGNETOHYDRO- DYNAMICS}

Turning now to the magnetohydrodynamic field, we see that for the two modes $\phi$ and $\psi$ of the Laplace operator, the fundamental solutions are both $(1 / 2 \pi) \log r$. In the next section we see that even though the fundamental solutions are the same for these two modes, they represent entirely different patterns of behavior.

In obtaining the fundamental solutions for the viscous-resistive mode, we consider here the case for which $\nu=\kappa$, leaving until later the case for arbitrary $\nu$ and $\kappa$. First let us consider the $\lambda^{\prime}$ mode which satisfies the equation

$$
\left(U+H_{x}\right)\left(\partial \lambda^{\prime} / \partial x\right)+H_{y}\left(\partial \lambda^{\prime} / \partial y\right)-\nu \nabla^{2} \lambda^{\prime}=0 .
$$

Unlike the Laplace equation, this equation is not isotropic, i.e., it is not invariant under a rotation of the coordinates. Consequently, its fundamental solution cannot be simply a function of $r$ as was that of the Laplace equation. However, this is a trivial shortcoming and is easily removed by introducing a simple exponential factor. Thus if we replace $\lambda^{\prime}$ by

$$
\tilde{\exp }\left\{\left[\left(U+H_{x}\right) x+H_{y} y\right] / 2 \nu\right\},
$$

we find that $\tilde{\lambda}$ satisfies

$$
\nabla^{2} \tilde{\lambda}-\left(W_{+}^{2} / 4 \nu^{2}\right) \tilde{\lambda}=0
$$

where

$$
W_{ \pm}=\left[\left(U \pm H_{x}\right)^{2}+H_{y}^{2}\right]^{\frac{1}{2}} .
$$

This equation is isotropic and we readily find that its fundamental solution is

$$
(1 / 2 \pi) K_{0}\left(r W_{+} / 2 v\right),
$$

where $K_{0}$ is the zeroth-order Bessel function of imaginary argument which behaves like a logarithm at the origin and like a negative exponential at infinity. Our fundamental solution for $\lambda^{\prime}$ is thus

$$
-\frac{1}{2 \pi \nu} \exp \left\{\left[\left(U+H_{x}\right) x+H_{\nu} y\right] / 2 \nu\right\} K_{0}\left(r W_{+} / 2 \nu\right) \text {. }
$$

Turning now to the other modes, we see that $\mu^{\prime}$ has the same fundamental solution as $\lambda^{\prime}$ and, by a simple change of signs, we find that the fundamental solution for $\chi$ and $\beta^{\prime}$ is

$$
-\frac{1}{2 \pi \nu} \exp \left\{\left[\left(U-H_{x}\right) x-H_{y} y\right] / 2 \nu\right\} K_{0}\left(r W_{-} / 2 \nu\right) .
$$

These fundamental solutions may be put in simple form by introducing the Reynolds numbers $R_{ \pm}=$ $r W_{\star} / 2 \nu$ and the polar angles $\theta_{\star}$ which are measured from the appropriate Alfvén directions. The fundamental solutions are all then given by

$$
-(1 / 2 \pi \nu) e^{R+\cos \theta \pm} K_{0}\left(R_{\perp}\right) \text {. }
$$

\section{BEHAVIOR OF THE FUNDAMENTAL MODES OF MAGNETOHYDRODYNAMICS}

Now that we have obtained the fundamental solutions for each of our modes, the next step is to examine in detail the field associated with each. First consider the $\phi$ and $\psi$ modes. For both, the fundamental solution is $(1 / 2 \pi) \log r$. Inserting this into the modal relationships for the $\phi$ and $\psi$ modes, we find that the velocity fields and the magnetic fields of these fundamental modes are made up of two basic patterns. The first of these is the twodimensional source shown in Fig. 1. The second is 
Fig. 1. Field pattern for potential sources and poles of the $\phi$ and $\psi$ modes.

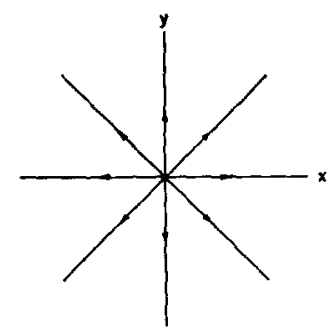

the vortex shown in Fig. 2. These same field patterns go by different names in magnetic terminology. The first becomes a pole and the second a current. In spite of the difference in terminology, the field patterns are the same.

For unit fundamental solution, we find that the $\phi$ mode consists of a velocity source of strength $U$ accompanied by a magnetic pole of strength $H_{x}$ and a current of strength $-H_{y}$. On the other hand, the $\psi$ mode consists of a velocity vortex of strength $-U$, a magnetic pole of strength $-H_{y}$ and a current of strength $-H_{x}$.

We thus see that for these two potential modes, the basic elements out of which the field is to be constructed are sources, vortices, poles and currents just as in the corresponding cases for "pure" fluid mechanics and "pure" magnetic field theory. And here, just as there, we may multiply these unit singularities by appropriate factors to obtain source strengths $Q$, vortex strengths $\Gamma$, pole strengths II, and current strengths $J$ which may then be distributed throughout the plane to obtain arbitrary field patterns. However, here these fields are intercoupled. If we use a source of strength $Q$, it must be accompanied by a pole of strength $Q H_{x} / U$ and a current of strength $-Q H_{y} / U$. Correspondingly, a vortex of strength $\Gamma$ must be accompanied by a pole of strength $\Gamma H_{y} / U$ and a current of strength $-\Gamma H_{x} / U$. Because of the symmetry in the roles of the velocity and the magnetic field, we may construct fundamental modes giving a pure pole and a pure current just as the above give pure sources and pure vortices. In this reversed role, the pole and the current will each be accompanied by attendant sources and vortices.

FIG. 2. Field pattern for potential vortices and currents of the $\phi$ and $\psi$ modes.
Fig. 3. Field pattern of viscous-resistive sources and poles of the $\lambda^{\prime}$ and $\mu^{\prime}$ modes with $\nu=\kappa$.

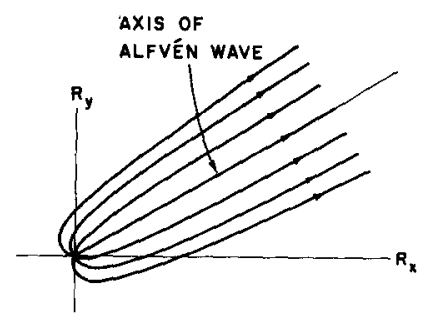

In the above analysis, we have established a tie-in between our present method of fundamental modes and the already familiar concepts of fluid mechanics and magnetic theory. Now let us push into less well known territory and investigate the viscous-resistive modes for $\nu=\kappa$.

For the $\lambda^{\prime}$ and $\mu^{\prime}$ modes the fundamental solution is

$-\frac{1}{2 \pi \nu} \exp \frac{\left(U+H_{x}\right) x+H_{y} y}{2 \nu} K_{0}\left\{\frac{r\left[\left(U+H_{x}\right)^{2}+H_{y}^{2}\right]^{\frac{1}{3}}}{2 \nu}\right\}$.

If we substitute this into the modal relationships for $\lambda^{\prime}$ and $\mu^{\prime}$ in Table II, we find that two intriguing new patterns make their appearance for the velocity and magnetic fields. These are shown in Figs. 3 and 4 . In the first of these, the fluid emerges sourcelike from the origin and when it has traveled out to a Reynolds number of order unity, it turns in a very educated way so as to flow in the direction of the Alfven wave whose angle is $\tan ^{-1}\left[H_{y} /\left(U+H_{x}\right)\right]$. In the second, the fluid rotates as a vortex near the origin, but out at unit Reynolds number, it develops a bulge and the circulation carries fluid out along the Alfvén wave and back again.

In the case of the $\lambda^{\prime}$ mode, the fundamental solution corresponds to a viscous resistive source at the origin of strength +1 accompanied by a viscous resistive pole of strength -1 . The magnetic field pattern of this pole is the same as the velocity pattern of the source. The $\mu^{\prime}$ fundamental solution consists of a viscous-resistive vortex of strength +1 accompanied by a viscous resistive current having the same field pattern and a strength of -1 .

The patterns for the $\chi^{\prime}$ and $\beta^{\prime}$ modes are obtained from those of the $\lambda^{\prime}$ and $\mu^{\prime}$ modes by a change of the signs of the magnetic field. This shifts the axis of the singularities from one Alfven direction to the

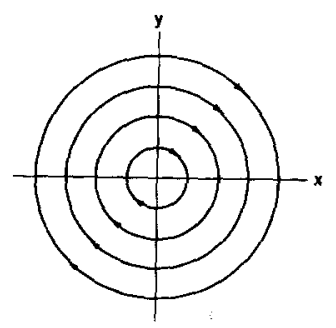

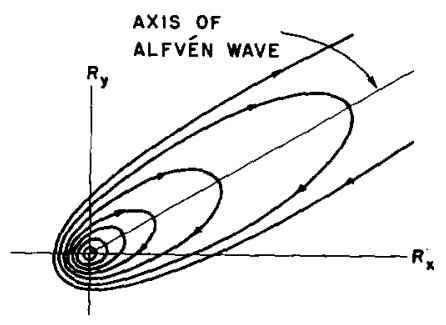

FiG. 4. Field pattern of viscous-resistive vortices and currents of the $\lambda^{\prime}$ and $\mu^{\prime}$ modes with $\nu=\kappa$. 


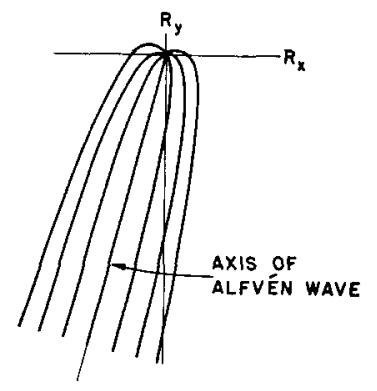

FIg. 5. Field pattern of viscous-resistive sources and poles of the $\chi^{\prime}$ and $\beta^{\prime}$ modes with $\nu=\kappa$.

other. The resulting field patterns are shown in Figs. 5 and 6 . For these solutions the strengths of the sources, poles, vortices and currents are all +1 .

Now that we have obtained the fields of the viscous-resistive singularities, the question immediately arises: Why do they behave as they do? To answer this question, let us go back to our original equations. The most characteristic feature of the viscous resistive mode is the fact that the pressure is zero. If we use this fact, we can rewrite the two momentum equations (with the help of the equations $\partial u / \partial x+\partial v / \partial y=0$ and $\partial h_{x} / \partial x+$ $\left.\partial h_{y} / \partial y=0\right)$ in the form

$-U \frac{\partial v}{\partial y}+\nu\left(\frac{\partial^{2} v}{\partial x \partial y}-\frac{\partial^{2} u}{\partial y^{2}}\right)+H_{x} \frac{\partial h_{y}}{\partial y}-H_{y} \frac{\partial h_{x}}{\partial y}=0$,

$U \frac{\partial v}{\partial x}+\nu\left(-\frac{\partial^{2} v}{\partial x^{2}}+\frac{\partial^{2} u}{\partial x \partial y}\right)-H_{x} \frac{\partial h_{y}}{\partial x}+H_{y} \frac{\partial h_{x}}{\partial x}=0$.

These can be integrated to give the single equation

$$
\nu(\partial v / \partial x-\partial u / \partial y)-H_{y} h_{x}+H_{x} h_{y}-U v=0,
$$

which, together with the equations

$$
\begin{gathered}
\kappa\left(\partial h_{y} / \partial x-\partial h_{x} / \partial y\right)-H_{y} u+H_{x} v-U h_{y}=0, \\
\partial u / \partial x+\partial v / \partial y=0 \\
\partial h_{x} / \partial x+\partial h_{y} / \partial y=0
\end{gathered}
$$

determines our viscous-resistive modes.

First let us consider the $\lambda^{\prime}$ mode in which the velocity field emerges from a source and the magnetic

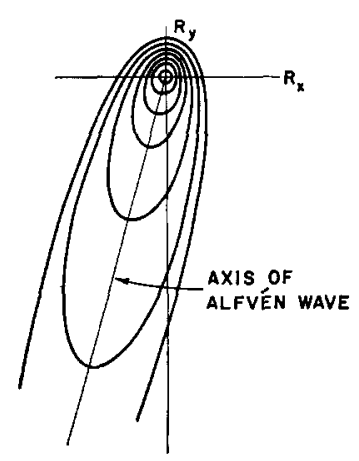

Frg. 6. Field pattern of viscous-resistive vortices and currents of the $\chi^{\prime}$ and $\beta^{\prime}$ modes with $y=\kappa$. field goes in to a pole. Near the singularity these fields are radial and if we insert such radial fields into the first two of the above modal equations, we find that their interaction with the gross fields, $U, H_{x}, H_{y}$ produces rotations $\partial v / \partial x-\partial u / \partial y$ and $\partial h_{y} / \partial x-\partial h_{x} / \partial y$, that turn the velocity and magnetic fields so that they point along the Alfven direction $\tan ^{-1}\left[H_{y} /\left(U+H_{x}\right)\right]$ as shown in Fig. 3 . Similar turning actions occur for the other modes. These turning actions are actions of diffusion. They are the way in which diffusion manifests itself in these fields.

It is interesting to note that in the case of the velocity field, this turning results from an interaction both of the velocity field with itself, $-U v$, and of the magnetic field with itself, $-H_{y} h_{x}+H_{x} h_{y}$, whereas the turning of the magnetic field results from an interaction of one with the other, $-H_{y} u+$ $H_{x} v-U h_{y}$. It is for this reason that in purely viscous flows without a magnetic field the viscous singularities still distort and form downstream wakes. However, the reverse will not be true for magnetic fields in resistive fluids for which the velocity is zero.

Let us see what happens to the above singularities when $\nu$ and $k$ are zero. In this case, the two factored operators

$$
\left(U+H_{x}\right) \frac{\partial}{\partial x}+H_{y} \frac{\partial}{\partial y}-\nu \nabla^{2}
$$

and

$$
\left(U-H_{x}\right) \frac{\partial}{\partial x}-H_{y} \frac{\partial}{\partial y}-\nu \nabla^{2}
$$

degenerate into the Alfvén wave operators. When this happens, two of the above fundamental modes must degenerate into the fundamental modes of the Alfven waves and the other two must in some way disappear. In Figs. 3-6, we have used the Reynolds numbers $x W_{ \pm} / 2 \nu$ and $y W_{+} / 2 \nu$ as coordinates. If we now use $x$ and $y$ as coordinates, and let $\nu$ (and $\kappa$ ) approach 0 , this will shrink the patterns of Figs. 3-6 until they coincide with the Alfvén lines. The sources of Figs. 3 and 5 will focus their outward flowing fluid into an ever narrower jet until in the limit the jet is concentrated along the Alfvén line. The velocities will become infinite, but the integral of their flux will remain unity. In our integral operator representation, this state of affairs can be represented by a distribution of Dirac functions along the Alfvén line. This distribution has unit cross-sectional strength or equivalently unit strength per unit length. However, this is exactly what we found earlier for the fundamental solutions 
for single waves. Consequently, it is the source-pole modes, $\lambda^{\prime}$ and $\chi^{\prime}$, that degenerate into the Alfvén waves as $\nu, \kappa \rightarrow 0$.

What about the vortex-current modes, $\mu^{\prime}$ and $\beta^{\prime}$ ? As the patterns of Figs. 5 and 6 shrink with decreasing $\nu$ and $\kappa$, the velocity at any given radius decreases until in the limit the velocity field is zero everywhere except at the origin. Thus in a "perfect" fluid ( $\nu=\kappa=0)$, the viscous resistive type of vortex is unable to transmit any of its rotation to the surrounding fluid. The $\mu^{\prime}$ and $\beta^{\prime}$ fundamental modes thus disappear when $\nu$ and $\kappa$ approach zero.

We may now summarize our findings about the behavior of the fundamental solutions for our six modes. Two of these modes have as fundamental singularities the conventional sources, vortices, poles, and currents of potential theory. Here, these singularities are coupled together in such a way that only two independent combinations or modes of these four singularities exist. In addition to these two potential modes, there are four viscous-resistive modes and for the case $\nu=\kappa$ these, too, have as fundamental singularities coupled sources, vortices, poles, and currents. However, the fields of these latter singularities exhibit an unusual distortion which takes place along one or the other of the Alfvén directions. These distortions are such that these singularities may actually be thought of as being diffuse Alfvén waves. These waves diffuse independently of each other. They show close similarity to the diffusing wakes of viscous flows and, in fact, they have taken over entirely the functions of these viscous wakes. The latter cease to exist in magnetohydrodynamics for this case in which $\nu=\kappa$. Strictly, it is the source and pole singularities rather than the vortex and current singularities that are the diffuse Alfvén waves. When we allow $\nu$ and $k$ to go to zero, these are the singularities that become the true Alfvén waves. However, the vortex and current singularities represent legitimate diffuse waves of a kind which can no longer be excited when $\nu, \kappa=0$, but which provide a degree of freedom required to meet the boundary conditions of real fluids where $\nu=\kappa \neq 0$. The above results are summarized in quantitative form in Table III.

\section{FUNDAMENTAL MODES FOR GENERAL VALUES OF $\nu$ AND *}

Thus far we have considered fundamental modes for the case in which $\nu$ and $\kappa$ are equal. We now turn our attention to the intriguing question of what happens for arbitrary values $\nu$ and $\kappa$. Not only are these cases interesting because of their wider applicability, but also because they shed light on the following question. When $\nu=\kappa$, the viscousresistive factor of the system operator itself factors into two second-order operators. These lead to the result that the two Alfven waves diffuse independently and that this diffusion is analogous to that of ordinary viscous wakes. However, when $\nu$ is not equal to $k$, no such factoring is possible and we must completely revise our way of sorting out the independent modes of the system. This has deep-seated consequences in the analysis and we shall find that a significant change is taking place

TABLE III. Fundamental modes for the case $\nu=\kappa$.

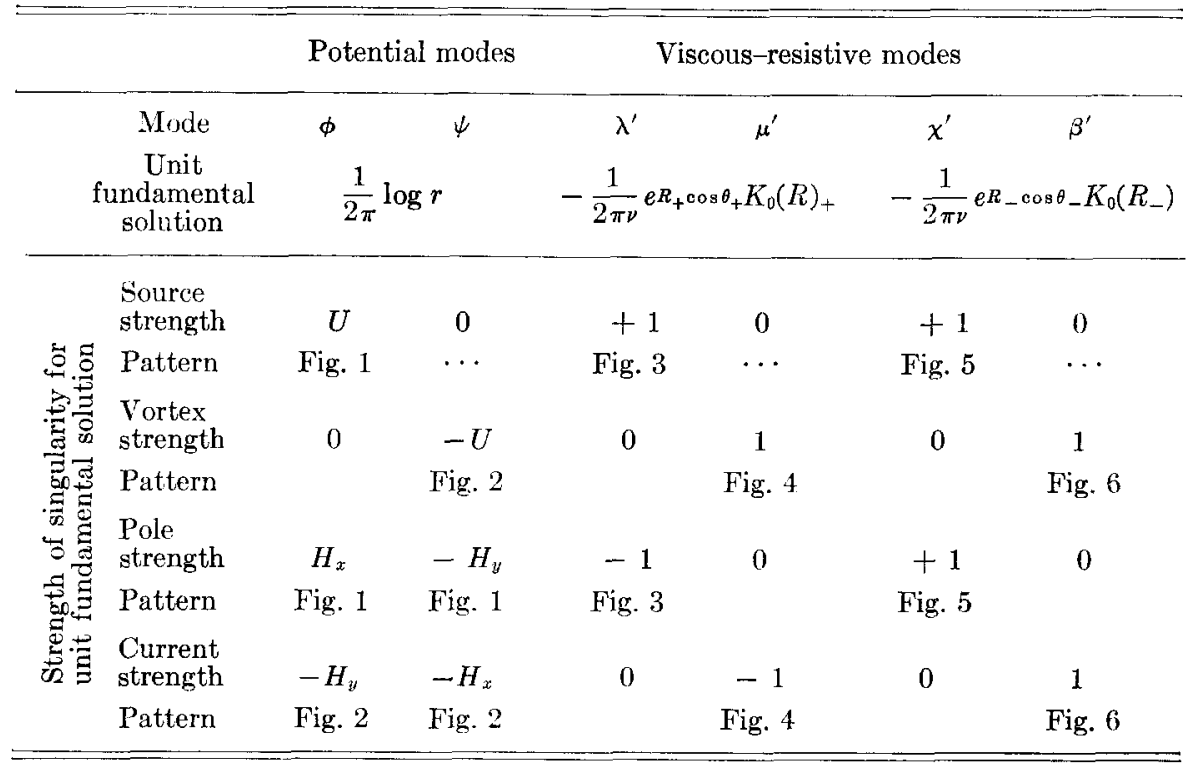




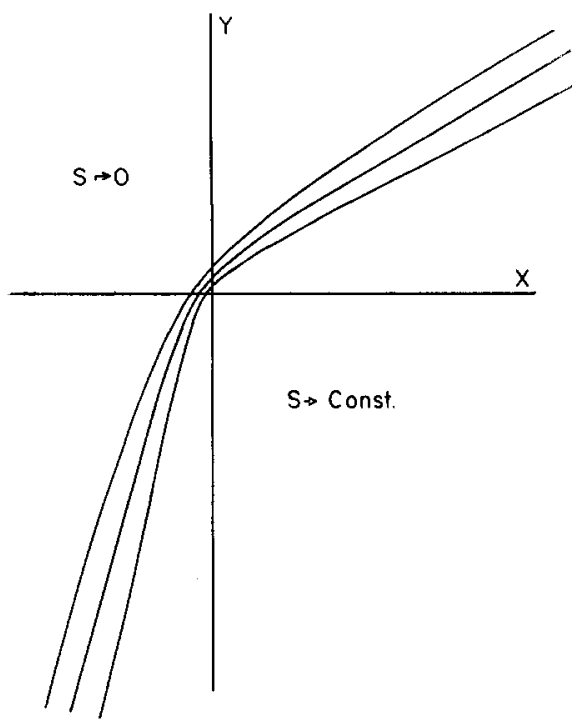

FIG. 7. Field pattern of the fundamental solution $S$ for the full viscous-resistive mode.

in the physical processes. By observing what happens when an operator becomes unfactorizable, we shall be able to obtain an insight into the more general meaning of unfactorizability and what it signifies physically.

For general values of $\nu$ and $\kappa$, the unfactorizable viscous-resistive operator is

$\left(U \frac{\partial}{\partial x}-\nu \nabla^{2}\right)\left(U \frac{\partial}{\partial x}-\kappa \nabla^{2}\right)-\left(H_{x} \frac{\partial}{\partial x}+H_{y} \frac{\partial}{\partial y}\right)^{2}$.

In Sec. $V$ we have obtained specific expressions for the modes of this operator in terms of the four modal variables $\lambda, \mu, \chi$, and $\beta$. However, we have not as yet obtained the fundamental solution for this fourth-order operator. Our present task, therefore, is to obtain this solution and to interpret the new concepts that will emerge when this solution is substituted into the modal relations.

In our earlier work, we went through the process of converting the above equation and its associated modal relations to the much simpler form they assume when $\nu=\kappa$. This was not simply a matter of putting $\nu=k$. The above operator split into two factors and these yielded two second-order equations with two fundamental solutions. The modal relations became of first order. And it was in this simpler form that we found the fundamental solutions given in the preceding section for $\nu=\kappa$. In contrast, when $\nu \neq \kappa$, there is only one fundamental solution and it obeys a fourth-order equation and the modal relations are of third order.

This drastic difference in form between the two cases $\nu=\kappa$ and $\nu \neq \kappa$ requires that we first convert our solutions for $\nu=\kappa$ into the form for use when $\nu \neq \kappa$ and then proceed to solve the case for $\nu \neq \kappa$.

For convenience, let us designate the two OseenAlfvén operators of our $\nu=\kappa$ case by $O_{+}$and $O_{-}$:

$$
\begin{aligned}
& O_{+} \equiv\left(U+H_{x}\right) \frac{\partial}{\partial x}+H_{y} \frac{\partial}{\partial y}-\nu \nabla^{2 \bar{z}} \\
& O_{-} \equiv\left(U-H_{x}\right) \frac{\partial}{\partial x}-H_{y} \frac{\partial}{\partial y}-\nu \nabla^{2}
\end{aligned}
$$

Also let us designate by $S_{+}$and $S_{-}$the two fundamental solutions of these operators:

$$
\begin{aligned}
& S_{+}=-\frac{1}{2 \pi \nu} \exp \left[\frac{\left(U+H_{x}\right) x+H_{y} y}{2 \nu}\right] K_{0}\left(\frac{r W_{+}}{2 \nu}\right), \\
& S_{-}=-\frac{1}{2 \pi \nu} \exp \left[\frac{\left(U-H_{x}\right) x-H_{y} y}{2 \nu}\right] K_{0}\left(\frac{r W_{-}}{2 \nu}\right) .
\end{aligned}
$$

If $\delta$ is the Dirac function with singularity at the origin, the equations that these solutions satisfy are $O_{+} S_{+}=\delta$ and $O_{-} S_{-}=\delta$. Now we would like to convert these two solutions into a single fundamental solution $S$ for the fourth-order operator $O \equiv O_{+} O_{-}$. This solution satisfies the equation $O S=\delta$, which can be written $O_{+} O_{-} S=\delta$. First let us invert the operator $O_{+}$, giving $O_{-} S=O_{+}^{-1} \delta$. But we know that $O_{+}^{-1} \delta=S_{+}$so we have $O_{-} S=S_{+}$. Next we invert $O_{-}$giving $S=O_{-}^{-1} S_{+}$. However, we know that the integral kernel for the inverse operator $O_{-}^{-1}$ is just the fundamental solution $S_{-}$. Consequently the desired fundamental solution $S$, valid for $\nu=\kappa$, is given by the integral

$S(x, y)=\int_{-\infty}^{\infty} \int_{-\infty}^{\infty} S_{-}\left(x-x^{\prime}, y-y^{\prime}\right) S_{+}\left(x^{\prime}, y^{\prime}\right) d x^{\prime} d y^{\prime}$,

which is just the convolution $S_{-} * S_{+}$.

The physical interpretation of this result is that we may obtain the fundamental solution $S$ by distributing singularities of type $S_{-}$throughout the plane with a density distribution of $S_{+}$. Because of the symmetry of the convolution, we may equally well distribute $S_{+}$singularities with a density $S_{-}$.

Since we have definite expressions for $S_{+}$and $S_{-}$, we may use them to compute $S$. In Fig. 7 we have plotted a set of contours for $S$ for a typical orientation of the magnetic field. This solution displays two branches which, as might be expected, run out along each of the Alfven directions. These come from the weighted distributions of $S_{+}$and $S_{-}$ which each run out along one or the other of the Alfvén directions.

Next let us turn to the problem of relating the third-order modal relationships used with $S$ to the first-order modal relationships used with $S_{+}$and $S_{-}$. 
If we examine the former, which are given in Sec. V, we see that when $\nu=\kappa$, they can be written in the form

$$
\begin{aligned}
u=-h_{x}=\left(U+H_{x}-\nu \frac{\partial}{\partial x}\right) & \\
\cdot & {\left[\left(U-H_{x}\right) \frac{\partial}{\partial x}-H_{y} \frac{\partial}{\partial y}-\nu \nabla^{2}\right] \lambda, } \\
v=-h_{\nu}=\left(H_{y}-\nu \frac{\partial}{\partial y}\right) & \\
\cdot & {\left[\left(U-H_{x}\right) \frac{\partial}{\partial x}-H_{y} \frac{\partial}{\partial y}-\nu \nabla^{2}\right] \lambda, }
\end{aligned}
$$

with similar expressions for the $\mu, \chi$, and $\beta$ modes. Correspondingly, the first-order relationships for use with $S_{+}$and $S_{-}$are

$$
\begin{aligned}
& u=-h_{x}=\left(U+H_{x}-\nu \frac{\partial}{\partial x}\right) \lambda^{\prime}, \\
& v=-h_{y}=\left(H_{\nu}-\nu \frac{\partial}{\partial y}\right) \lambda^{\prime},
\end{aligned}
$$

with similar expressions for $\mu^{\prime}, \chi^{\prime}$ and $\beta^{\prime}$ (see Table II).

We see that the $\lambda$ and $\mu$ modal relations are the same as the $\lambda^{\prime}$ and $\mu^{\prime}$ modal relations except that they contain the additional factor

$$
O_{\sim}=\left(U-H_{x}\right) \frac{\partial}{\partial x}-H_{y} \frac{\partial}{\partial y}-\nu \nabla^{2} .
$$

Similarly the $\chi$ and $\beta$ modal relations are the same as the $\chi^{\prime}$ and $\beta^{\prime}$ modal relations except that they contain the additional factor $O_{+}$.

Next, let us relate this result to the preceding result that $S$ is the convolution of $S_{+}$and $S_{-}$. The fundamental singularities are obtained either by substituting the fundamental solution $S$ into the above expressions for $\lambda, \mu, \chi$ and $\beta$ or by substituting $S_{+}$and $S_{-}$into the corresponding expressions for $\lambda^{\prime}, \mu^{\prime}$ and $\chi^{\prime}, \beta^{\prime}$. Earlier, we found that when $O_{-}$ acts upon the convolutions $S_{-} * S_{+}$it removes $S_{-}$, that is, $O_{-} S=O_{-}\left(S_{-} * S_{+}\right)=S_{+}$. Similarly $O_{+} S=O_{+}\left(S_{-} * S_{+}\right)=S_{-}$. Thus we see that the factors $O_{-}$and $O_{+}$in the $\lambda, \mu$ and $\chi, \beta$ modal relations will remove $S_{-}$or $S_{+}$from $S$ and will lead to the same expressions as obtained for the $\lambda^{\prime}, \mu^{\prime}, \chi^{\prime}$, and $\beta^{\prime}$ modes. This establishes the complete correspondence between the two sets of relations.

Now that we have completed the task of converting our results for $\nu=\kappa$ into the form for use when $\nu \neq \kappa$, we are ready to seek fundamental solutions $S$ for arbitrary $\nu$ and $\kappa$. Such solutions are not simple functions. They satisfy a rather complex fourth-order equation. As yet, it has not been possible to obtain exact solutions in closed form for $S$ and indeed it is doubtful that exact solutions can ever be found in simple form. However, it has been possible to map out in detail the complete characteristics of these solutions so that we may explore rather thoroughly the new phenomena that appear when $\nu$ and $\kappa$ are no longer equal.

The equation which $S$ must satisfy is

$$
\begin{aligned}
\left(U \frac{\partial}{\partial x}-\nu \nabla^{2}\right)\left(U \frac{\partial}{\partial x}-\kappa \nabla^{2}\right) S & \\
& -\left(H_{x} \frac{\partial}{\partial x}+H_{y} \frac{\partial}{\partial y}\right)^{2} S=\delta .
\end{aligned}
$$

First, let us examine the behavior of $S$ near the origin so that we may determine the nature of the singularities that occur there. Very near the origin, the derivatives of highest order will be controlling. This means that we should first examine solutions of the equation $\nu \kappa \nabla^{4} S=\delta$, which retains only these highest derivatives. The fundamental solution for this equation is $S=r^{2}(\log r-2) / 8 \pi \nu$. Turning now to the modal relationships in which this fundamental solution is used (see Sec. V) we see that if we retain the highest derivatives, these relationships will take the form given in Table IV.

By direct substitution of the fundamental solution $S=r^{2}(\log r-2) / 8 \pi \nu \kappa$ into these modal relationships, we find that the four viscous-resistive modes continue to have fundamental singularities which are sources, poles, vortices, and currents. Furthermore, the strengths of these singularities continue to be as given in Table III. Thus we see that the

\begin{tabular}{|c|c|c|c|c|}
\hline & $\lambda$ & $\mu$ & $\chi$ & $\beta$ \\
\hline$u$ & $\nu_{\kappa} \frac{\partial}{\partial x} \nabla^{2} \lambda$ & $-\nu_{\kappa} \frac{\partial}{\partial y} \nabla^{2} \mu$ & $\nu_{\kappa} \frac{\partial}{\partial x} \nabla^{2} \chi$ & $-\nu_{k} \frac{\partial}{\partial y} \nabla^{2} \beta$ \\
\hline$v^{\prime \prime}$ & $\nu_{\kappa} \frac{\partial}{\partial y} \nabla^{2} \lambda$ & $\nu \kappa \frac{\partial}{\partial x} \nabla^{2} \mu$ & $\nu_{k} \frac{\partial}{\partial y} \nabla^{2} \chi$ & $\nu \kappa \frac{\partial}{\partial x} \nabla^{2} \beta$ \\
\hline$h_{x}$ & $-\mu^{\mu} \frac{\partial}{\partial x} \nabla^{\lambda} \lambda$ & $\nu_{\kappa} \frac{\partial}{\partial y} \nabla^{2} \mu$ & $\nu_{\kappa} \frac{\partial}{\partial x} \nabla^{2} \chi$ & $-\nu \kappa \frac{\partial}{\partial y} \nabla^{2} \beta$ \\
\hline$h_{y}$ & $-\nu \kappa \frac{\partial}{\partial y} \nabla^{2} \lambda$ & $-\nu \kappa \frac{\partial}{\partial x} \nabla^{2} \mu$ & $\nu_{\kappa} \frac{\partial}{\partial y} \nabla^{2} \chi$ & $\nu \kappa \frac{\partial}{\partial x} \nabla^{2} \beta$ \\
\hline
\end{tabular}
condition $\nu \neq k$, and the nonsplitting of the viscousresistive operator into factors, has nothing to do with the field pattern of the four fundamental field singularities in the immediate vicinity of the origin.

In our earlier work for $\nu=\kappa$, we saw that the viscous-resistive singularities resemble conventional

TABLF IV. Highest-order derivatives in the viscous-resistive modal relationships for arbitrary $\nu$ and $\kappa$. 
singularities near the origin, but farther out they take on a characteristic distortion in the direction of the Alfvén waves (see Figs. 3-6). We now ask what happens to these distortions when $\nu$ and $\kappa$ are not equal. Such distortions are controlled by lower order derivatives than we have so far retained. If we keep derivatives of one order lower, the distortions begin at a radial Reynolds number of order unity, just as they did before, but now we must use $2 \nu \kappa /(\nu+\kappa)$ as the diffusion coefficient in place of $\nu$ or $\kappa$. Unfortunately, these next-lower-order derivatives do not tell us the directions in which the distortions occur. To answer this question, we must go to even lower order derivatives. However our expressions all contain only three orders of derivatives and this means that we must deal with the full expressions and we are back to our original difficulties. However, with the foregoing information at hand, we may now attack the problem from a slightly different angle.

We have seen above that the effective diffusion coefficient near the singularity is $2 \nu \kappa /(\nu+\kappa)$. In addition to this parameter, let us introduce the nondimensional quantity $(\nu-\kappa) /(\nu+\kappa)$, which measures the differential diffusion of $\nu$ and $\kappa$. We now divide our analysis into the following parts. First we investigate what happens when $\nu$ and $\kappa$ are not greatly different so that $(\nu-\kappa) /(\nu+\kappa)$ is small. Next we investigate what happens when $\nu$ and $\kappa$ are quite different. Since $\nu$ and $\kappa$ are always positive this will occur when $(\nu-\kappa) /(\nu+\kappa)$ is near either +1 or -1 .

Let us start with the case for which $(\nu-\kappa) /(\nu+\kappa)$ is small. We impose the double condition: we neglect second-order terms in $(\nu-\kappa) /(\nu+\kappa)$ but only for those derivatives which are of lowest rank. This stratagem permits us to determine the distortion of the singularities when $\nu$ and $\kappa$ are not greatly different. Under this double condition, the equation for $S$ can be written in the form

$$
\begin{aligned}
& {\left[\left(U+H_{x}\right) \frac{\partial}{\partial x}+H_{\nu} \frac{\partial}{\partial y}-\frac{2 \nu \kappa}{\nu+\kappa} \nabla^{2}\right]} \\
& \quad\left[\left(U-H_{x}\right) \frac{\partial}{\partial x}-H_{y} \frac{\partial}{\partial y}-\frac{2 \nu \kappa}{\nu+\kappa} \nabla^{2}\right] S=\frac{4 \nu \kappa \delta}{(\nu+\kappa)^{2}} .
\end{aligned}
$$

Since this equation has exactly the same form as it did when $\nu=\kappa$, the fundamental solution $S$ must be the same. All we need do is replace $\nu$ by $2 \nu_{\kappa} /(\nu+\kappa)$ and multiply the solution by $4 \nu \kappa /(\nu+\kappa)^{2}$ (since this factor appears in front of $\delta$ ). $S$ will continuc to have the same behavior that is shown typically in Fig. 7.
Turning now to the modal relationships to be used with this solution, we find that under the same dual condition, these relations can be written:

\section{Mode}

$$
\begin{aligned}
& u=\frac{(\nu+\kappa)^{2}}{4 \nu \kappa}\left(U+H_{x}-\frac{2 \nu \kappa}{\nu+\kappa} \frac{\partial}{\partial x}\right) O_{-} \lambda \\
& -\frac{\nu-\kappa}{2} \frac{\partial}{\partial y}\left[H_{y} \frac{\partial}{\partial x}-\left(U+H_{x}\right) \frac{\partial}{\partial y}\right] \lambda, \\
& v=\frac{(\nu+\kappa)^{2}}{4 \nu \kappa}\left(H_{y}-\frac{2 \nu \kappa}{\nu+\kappa} \frac{\partial}{\partial y}\right) O_{-} \lambda \\
& +\frac{\nu-\kappa}{2} \frac{\partial}{\partial x}\left[H_{y} \frac{\partial}{\partial x}-\left(U+H_{x}\right) \frac{\partial}{\partial y}\right] \lambda, \\
& h_{x}=-\frac{(\nu+\kappa)^{2}}{4 \nu \kappa}\left(U+H_{x}-\frac{2 \nu \kappa}{\nu+\kappa} \frac{\partial}{\partial x}\right) O_{-} \lambda \\
& -\frac{\nu-\kappa}{2} \frac{\partial}{\partial y}\left[H_{y} \frac{\partial}{\partial x}-\left(U+H_{x}\right) \frac{\partial}{\partial y}\right] \lambda,
\end{aligned}
$$

$$
\begin{gathered}
h_{y}=-\frac{(\nu+\kappa)^{2}}{4 \nu \kappa}\left(H_{y}-\frac{2 \nu \kappa}{\nu+\kappa} \frac{\partial}{\partial y}\right) O_{-\lambda} \\
+\frac{\nu-\kappa}{2} \frac{\partial}{\partial x}\left[H_{y} \frac{\partial}{\partial x}-\left(U+H_{x}\right) \frac{\partial}{\partial y}\right] \lambda . \\
u \text { Mode }
\end{gathered}
$$

\section{u Mode}

$$
\begin{aligned}
& u=\frac{\nu+\kappa}{2} \frac{\partial}{\partial y} O_{-\mu} \\
& \quad+\frac{\nu-\kappa}{2} \frac{\partial}{\partial y}\left[\left(U+H_{x}\right) \frac{\partial}{\partial x}+H_{y} \frac{\partial}{\partial y}\right] \mu,
\end{aligned}
$$

$$
\begin{aligned}
v=-\frac{\nu+\kappa}{2} & \frac{\partial}{\partial x} O_{-\mu} \\
& \quad-\frac{\nu-\kappa}{2} \frac{\partial}{\partial x}\left[\left(U+H_{x}\right) \frac{\partial}{\partial x}+H_{y} \frac{\partial}{\partial y}\right] \mu,
\end{aligned}
$$$$
h_{x}=-\frac{\nu+\kappa}{2} \frac{\partial}{\partial y} O_{-} \mu
$$$$
+\frac{\nu-\kappa}{2} \frac{\partial}{\partial y}\left[\left(U+H_{x}\right) \frac{\partial}{\partial x}+H_{y} \frac{\partial}{\partial y}\right] \mu,
$$$$
h_{y}=\frac{\nu+\kappa}{2} \frac{\partial}{\partial x} O_{-} \mu
$$

$$
-\frac{\nu-\kappa}{2} \frac{\partial}{\partial x}\left[\left(U+H_{x}\right) \frac{\partial}{\partial x}+H_{y} \frac{\partial}{\partial y}\right] \mu .
$$

\section{$x$ Mode}

$$
\begin{aligned}
u=\frac{(\nu+\kappa)^{2}}{4 \nu \kappa} & \left(U-H_{x}-\frac{2 \nu \kappa}{\nu+\kappa} \frac{\partial}{\partial x}\right) O_{+} \chi \\
& +\frac{\nu-\kappa}{2} \frac{\partial}{\partial y}\left[H_{y} \frac{\partial}{\partial x}+\left(U-H_{x}\right) \frac{\partial}{\partial y}\right] \chi,
\end{aligned}
$$




$$
\begin{aligned}
& y=-\frac{(\nu+\kappa)^{2}}{4 \nu \kappa}\left(H_{y}+\frac{2 \nu \kappa}{\nu+\kappa} \frac{\partial}{\partial y}\right) O_{+} \chi \\
& -\frac{v-\kappa}{2} \frac{\partial}{\partial x}\left[H_{y} \frac{\partial}{\partial x}+\left(U-H_{x}\right) \frac{\partial}{\partial y}\right] x, \\
& h_{x}=\frac{(\nu+\kappa)^{2}}{4 \nu \kappa}\left(U-H_{z}-\frac{2 v \kappa}{\nu+\kappa} \frac{\partial}{\partial x}\right) O_{+} \chi \\
& -\frac{v-\kappa}{2} \frac{\partial}{\partial y}\left[H_{y} \frac{\partial}{\partial x}+\left(U-H_{x}\right) \frac{\partial}{\partial y}\right] x, \\
& h_{l y}=-\frac{(\nu+\kappa)^{2}}{4 \nu \kappa}\left(H_{y}-\frac{2 \nu \kappa}{\nu+\kappa} \frac{\partial}{\partial y}\right) O_{+\chi} \\
& +\frac{\nu-\kappa}{2} \frac{\partial}{\partial x}\left[H_{y} \frac{\partial}{\partial x}+\left(U-H_{x}\right) \frac{\partial}{\partial y}\right] \chi .
\end{aligned}
$$

\section{Mode}

$$
\begin{aligned}
& u=\frac{\nu+\kappa}{2} \frac{\partial}{\partial y} O_{+} \beta \\
& \quad+\frac{\nu-\kappa}{2} \frac{\partial}{\partial y}\left[\left(U-H_{x}\right) \frac{\partial}{\partial x}-H_{y} \frac{\partial}{\partial y}\right] \beta, \\
& v=-\frac{\nu+\kappa}{2} \frac{\partial}{\partial x} O_{+} \beta \\
& \quad-\frac{\nu-\kappa}{2} \frac{\partial}{\partial x}\left[\left(U-H_{x}\right) \frac{\partial}{\partial x}-H_{y} \frac{\partial}{\partial y}\right] \beta,
\end{aligned}
$$

$$
\begin{aligned}
h_{x} \quad \frac{\nu+\kappa}{2} & \frac{\partial}{\partial y} O_{+} \beta \\
& \quad-\frac{\nu-\kappa}{2} \frac{\partial}{\partial y}\left[\left(U-H_{x}\right) \frac{\partial}{\partial x}-H_{y} \frac{\partial}{\partial y}\right] \beta,
\end{aligned}
$$$$
h_{y}=-\frac{\nu+\kappa}{2} \frac{\partial}{\partial x} O_{+} \rho
$$$$
+\frac{\nu-\kappa}{2} \frac{\partial}{\partial x}\left[\left(U-H_{x}\right) \frac{\partial}{\partial x}-H_{y} \frac{\partial}{\partial y}\right] \beta \text {. }
$$

In these expressions, we are now using $O_{+}$and $O_{-}$ to represent the operators

$$
(U+H) \frac{\partial}{\partial x}+H_{u} \frac{\partial}{\partial y}-\frac{2 \nu \kappa}{\nu+\kappa} \nabla^{2}
$$

and

$$
\left(U-H_{x}\right) \frac{\partial}{\partial x}-H_{y} \frac{\partial}{\partial y}-\frac{2 \nu \kappa}{\nu+\kappa} \nabla^{2}
$$

These modal relationships contain a basic portion which is identical with the modal relationships for the case $\nu=\kappa$ except that $2 \nu \kappa /(\nu+\kappa)$ replaces $\nu$. This basic portion, when acting upon $S$; will lead to the same velocity and magnetic fields pictured in Figs. 3-6 with the same kinds of distortions.

In addition to the basic portion of these relationships, there is a part which contains terms that are of first order in the quantity $\nu-\kappa$. These are the terms that introduce for the first time the new features arising from the inequality of $\nu$ and $k$. To lay the groundwork for exploring these new features, let us recall that the fundamental solution $S$ is a convolution $S_{-} * S_{+}$of the two solutions $S_{+}$and $S_{-}$. Furthermore, that when $O_{+}$or $O_{-}$act upon the convolution, they remove the factor $S_{-}$ or $S_{+}$from the convolution. It is for this reason that even though the fundamental solution $S$ contains two branches (see Fig. 7), the basic portion of the operator containing $O_{+}$or $O_{-}$will remove one of these branches and will leave a field which is distorted along just one of the Alfvén directions. It is also for this reason that we may think of the basic diffusion of the viscous-resistive sources, vortices, poles and currents as taking place separately along each of the Alfvén directions.

In contrast to this situation, the first-order terms in $\nu-\kappa$ do not contain the factors $\mathrm{O}_{+}$or $\mathrm{O}_{-}$to remove one or the other of the branches of $S$. Consequently, the contribution of these terms will be felt along both Alfvén wave directions and also in the fan-shaped region between them.

Let us take a closer look at these new terms. In the $\lambda$ mode all of the new terms contain the factor $H_{y}(\partial \lambda / \partial x)-\left(U+H_{x}\right)(\partial \lambda / \partial y)$ which is the derivative in the direction normal to $\tan ^{-1}\left[H_{y} /\left(U+H_{x}\right)\right]$. This latter is the Alfvén direction of the principal axis of the $\lambda$ mode. This derivative appears in the $\lambda$ modal relations as a stream function for both the velocity field and the magnetic field. Consequently it will contribute fields which point along the lines of constant values of this derivative. A look at the fundamental solution of Fig. 7 shows that these new terms, with $\kappa \gg v$, cause fluid to be diverted out of the upper side of the velocity jet of Fig. 3 . This fluid, after leaving the jet, turns right around and flows back to encircle the origin. It is then carried out and back along the conjugate Alfvén direction and finally rejoins the main jet smoothly on the latter's underside. The net result of all of this is to increase the strength of the lower side of the main jet at the expense of the upper side and at the same time to create a negative vortex around the jet which bulges out along the Alfven direction conjugate to the axis of this, the $\lambda$ mode.

If $v$ is greater than $\kappa$, the fluid leaves the bottom of the jet, turns around and makes the reverse circuit to rejoin the jet smoothly on its topside. For the magnetic field, the behavior for both $k>v$ and $\nu>\kappa$ is much the same as the velocity field except that the direction of the field lines is reversed, since the positive source is replaced by a negative pole, and the field patterns for $\kappa>p$ 


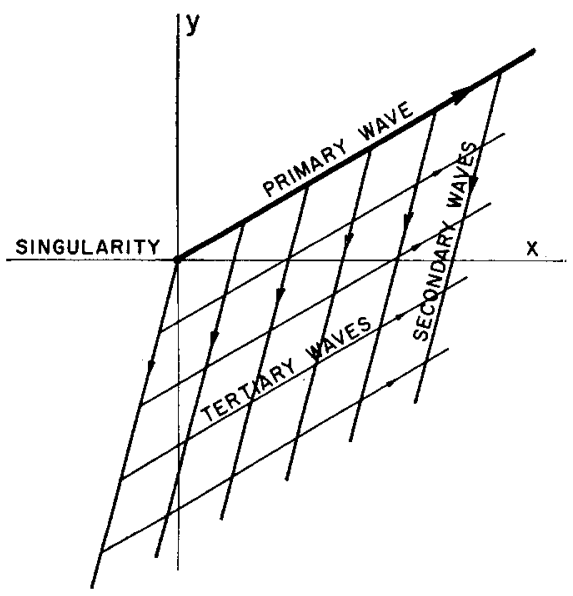

Fig. 8. Excitation of secondary and tertiary waves by differential diffusion when $\nu \neq \kappa$.

are interchanged with those for $\nu>\kappa$ and vice versa.

Turning now to the $\mu$ mode, a similar analysis shows that the following new features are added for this case. For $\nu>\kappa$, a portion of the fluid which is traveling outward on the underside of the negative of the vortex of Fig. 4 is diverted out of the vortex and turns to flow out and back along the conjugate Alfvén direction. It then makes a final turn to rejoin the main vortex flow on its topside. The next result of this is to create a countervortex around the main vortex, the countervortex having a bulge in the conjugate Alfvén direction.

When $\kappa>\nu$, the situation for the $\mu$ mode is much the same as for $\nu>\kappa$, except that the added vortex had the same sign as the main vortex rather than a reversed sign. As a result, the added flow leaves and rejoins the main flow smoothly without a turn. For the magnetic field, the field patterns are much the same as for the velocity field except that the cases $\nu>\kappa$ and $\kappa>\nu$ are interchanged. The behavior of the $\chi$ and $\beta$ modes can be obtained from that given above for the $\lambda$ and $\mu$ modes by changing the sign of the magnetic field, which interchanges the Alfven directions.

Now that we have seen what happens, it is appropriate to ask why it happens. Earlier we saw from the equations

$$
\begin{aligned}
& \nu\left(\frac{\partial v}{\partial x}-\frac{\partial u}{\partial y}\right)-H_{y} h_{x}+H_{x} h_{y}-U v=0, \\
& \kappa\left(\frac{\partial h_{y}}{\partial x}-\frac{\partial h_{x}}{\partial y}\right)-H_{y} u+H_{x} v-U h_{y}=0,
\end{aligned}
$$

that when the velocity and magnetic fields go into or out of the singularities, or circle around them, the interaction with the gross fields $U, H_{x}, H_{y}$ produces rotations $\partial v / \partial x-\partial u / \partial y$ and $\partial h_{y} / \partial x-\partial h_{x} / \partial y$ which turn the fields so as to distort them along one or the other of the Alfvén wave directions. When $\nu=\kappa$, it is possible to choose proper combinations of velocity and magnetic singularities such that these fields will turn and diffuse together along a single Alfvén wave direction. This means that in the case $\nu=\kappa$ each Alfvén wave will appear to diffuse separately.

However, when $\nu$ and $\kappa$ are not equal, the turning (and resulting diffusing) are not the same and the nice similarity between the two is upset. No longer will simple diffusing waves be possible. The differential diffusion of the primary wave excites waves of the other family into action. This will occur throughout the length of the primary wave, but will be most intense near the singularity which produces the primary wave. These secondary waves will travel across the fan in the wave direction which is complementary to that of the primary wave as shown in Fig. 8. These waves will in turn diffuse and will excite a set of tertiary waves which will travel parallel to the primary waves. This process will continue until the fan-shaped region is smoothly filled with a double set of diffusing waves. Because of the smoothness of the distribution and the lack of identity of single waves of either set, the resulting field will appear to be a diffuse wake emanating from the primary wave.

Let us continue with our analysis by now considering cases in which $\nu$ and $\kappa$ are quite different. Associated with each of these coefficients are the diffusion lengths $\nu / W_{*}$ and $k / W_{ \pm}$. These lengths are now greatly different. We have seen earlier that the four viscous-resistive singularities begin to distort at a radius of order $\nu \kappa /(\nu+\kappa) W_{ \pm}$. When $\nu$ and $\kappa$ are widely different, this distortion radius is equal to the smaller of the two diffusion lengths $\nu / W_{\text {s. }}$. and $\kappa / W_{ \pm}$.

If we begin by letting say $\nu$ go to zero, we find that a significant change takes place in our modes. The order of the system operator decreases from four to three and along with this goes a decrease in the number of modes. In this change the $\mu$ and $\beta$ modes become identical. Furthermore, the radius of distortion of the singularities shrinks to zero and their outer fields undergo important modifications.

Let us look at these changes in some detail. The equation for the fundamental solution with $\nu=0$ is

$$
\begin{aligned}
-{ }_{\kappa} U \frac{\partial}{\partial x} \nabla^{2} S+ & {\left[\left(U+H_{x}\right) \frac{\partial}{\partial x}+H_{y} \frac{\partial}{\partial y}\right] } \\
& \cdot\left[\left(U-H_{x}\right) \frac{\partial}{\partial x}-H_{y} \frac{\partial}{\partial y}\right] S=\delta .
\end{aligned}
$$


TABLE V. Highest-order derivatives in the viscous-resistive modal relationships for $\nu=0$.

\begin{tabular}{|c|c|c|c|}
\hline Mode & $\lambda$ & $\mu$ and $-\beta$ & $x$ \\
\hline & $-\kappa\left(U+H_{x}\right) \nabla^{2}$ & $-{ }_{\kappa} H_{\nu} \nabla^{2}$ & $-\kappa\left(U-H_{x}\right) \nabla^{2}$ \\
\hline$u$ & $+\kappa \frac{\partial}{\partial x}\left(H_{x} \frac{\partial}{\partial x}+H_{y} \frac{\partial}{\partial y}\right)$ & $+\kappa \frac{\partial}{\partial x}\left(H_{y} \frac{\partial}{\partial x}-H_{x} \frac{\partial}{\partial y}\right)$ & $-\kappa \frac{\partial}{\partial x}\left(H_{x} \frac{\partial}{\partial x}+H_{y} \frac{\partial}{\partial y}\right)$ \\
\hline$v$ & $\kappa \frac{\partial}{\partial x}\left(-H_{y} \frac{\partial}{\partial x}+H_{x} \frac{\partial}{\partial y}\right)$ & $\kappa \frac{\partial}{\partial x}\left(H_{x} \frac{\partial}{\partial x}+H_{y} \frac{\partial}{\partial y}\right)$ & $\kappa \frac{\partial}{\partial x}\left(H_{v} \frac{\partial}{\partial x}-H_{x} \frac{\partial}{\partial y}\right)$ \\
\hline$h_{x}$ & $\kappa \frac{\partial}{\partial x}\left(U \frac{\partial}{\partial x}\right)$ & $\kappa \frac{\partial}{\partial x}\left(-U \frac{\partial}{\partial y}\right)$ & $\kappa \frac{\partial}{\partial x}\left(-U \frac{\partial}{\partial x}\right)$ \\
\hline$h_{y}$ & $\kappa \frac{\partial}{\partial x}\left(U \frac{\partial}{\partial y}\right)$ & $\kappa \frac{\partial}{\partial x}\left(U \frac{\partial}{\partial x}\right)$ & $\kappa \frac{\partial}{\partial x}\left(-U \frac{\partial}{\partial y}\right)$ \\
\hline
\end{tabular}

To investigate the singularities themselves, we need retain only the highest derivatives, i.e. we need investigate $-{ }_{\kappa} U(\partial / \partial x) \nabla^{2} S=\delta$. To obtain the fundamental solution for this equation, we introduce the two functions $S_{1}$ and $S_{2}$ which satisfy $-\kappa \nabla^{2} S_{1}=\delta$ and $U\left(\partial S_{2} / \partial x\right)=\delta$. The solution for $S_{1}$ is $-(1 / 2 \pi \kappa) \log r$, and the solution for $S_{2}$ is a Dirac distribution along the positive $x$ axis having a strength of $1 / U$ per unit length. The desired solution for $S$ is the convolution of these two, i.e. $S_{1} * S_{2}$.

Turning now to the modal relationships in which this solution will be used, we put $\nu=0$ and retain only the highest-order derivatives. The resulting expressions are given in Table $\mathrm{V}$. These expressions are of two kinds. One contains the factor $\nabla^{2}$ and the other contains the factor $\partial / \partial x$. When these operators act upon the fundamental solution $S$, the factor $\nabla^{2}$ removes $S_{1}$ from the convolution $S_{1} * S_{2}$ and the factor $\partial / \partial x$ removes $S_{2}$. This leaves either a simple numerical factor multiplying $S_{2}$ or various combinations of first derivatives acting upon $S_{1}$. The numerical factor times $S_{2}$ corresponds to inviscid slipstreams trailing downstream from the singularity at the origin. The combination of first derivatives acting upon $S_{1}$ gives a set of sources, vortices, poles and currents. The strengths of all these singularities are given in Table VI.

These results do not agree with those of Table III and this is puzzling because we have found previously that the results of Table III should continue to be valid for general $\nu$ and $\kappa$. The resolution of this apparent discrepancy will come presently when we investigate the case for which $\nu$ is small but not zero.

The fields which we have just obtained are valid only in the immediate vicinity of the singularities. To see how they look at greater distances, we must retain lower-order derivatives. It has not been pos- sible to obtain exact solutions for these fields, but it has been possible to deduce the following facts about them.

In the case of the $\lambda$ mode, the inviscid slipstream, after emerging rearwardly from the singularity as a jet of zero width, spreads outward, turns and flows along the upper Alfven direction. This jet, which is originally of strength $\left(U+H_{x}\right) / U$, loses fluid to the surrounding flow so that its final strength is +1 . At great distances from the origin this jet, now diffuse, has the same pattern of that of Fig. 3 . The fluid which the jet loses flows in to the origin as a negative source of strength $-H_{x} / U$. In addition, the turning of the jet counteracts the diffuse outer vortex of strength $H_{y} / U$ so that no vortex is evident at great distances from the origin. The spreading and turning of the jet begins at a radius which is a small multiple of $\kappa / W_{+}$and the final transition to the outer flow of Fig. 3 is accomplished at a radius which is a large multiple of $\kappa / W_{+}$. The magnetic field undergoes less profound changes. Roughly, its field pattern is the same as it was when $\nu=\kappa$, except that the scale is increased. Finally, we may remark that for distances which are very large compared to $\kappa / W_{+}$, the $\lambda$ mode with $\nu=0$ and finite

TABLE VI. Strengths of the singularities for the various modes when $\boldsymbol{\nu}=0$.

\begin{tabular}{clccc}
\hline \hline Mode & \multicolumn{1}{c}{$\lambda$} & $\mu$ and $-\beta$ & $\chi$ \\
\hline & Source & $-H_{x} / U$ & $-H_{y} / U$ & $H_{x} / U$ \\
$\begin{array}{c}\text { Strengths } \\
\text { of } \\
\text { singularities } \\
\text { for } \\
\begin{array}{c}\text { unit } \\
\text { fundamental } \\
\text { solution }\end{array}\end{array}$ & Portex & $H_{y} / U$ & $-H_{x} / U$ & $-H_{y} / U$ \\
& $\begin{array}{l}\text { Current } \\
\text { slipstream } \\
\text { in }\end{array}$ & -1 & 0 & 1 \\
& U direction & -1 & 0 \\
\hline \hline
\end{tabular}


$\kappa$ has the same velocity and magnetic field patterns that it does for $\nu=\kappa$ except for a change in scale.

Now, let us turn to the problem of what is happening when $\nu$ is small compared to $\kappa$, but is not zero. In this case, all four modes are distinct, and the strengths of the singularities are those given in Table III. If we examine these singularities closely, we find that the following is happening.

In the case of the $\lambda$ mode, the velocity field emerges from a source at the origin and the magnetic field enters a pole there. As we have seen earlier, the equations

$$
\begin{aligned}
& \nu\left(\frac{\partial v}{\partial x}-\frac{\partial u}{\partial y}\right)-H_{y} h_{x}+H_{x} h_{y}-U v=0, \\
& \kappa\left(\frac{\partial h_{y}}{\partial x}-\frac{\partial h_{x}}{\partial y}\right)-H_{y} u+H_{x} u-U h_{y}=0,
\end{aligned}
$$

show that these fields upon interacting with the gross fields $U, H_{x}, H_{y}$ experience rotations $\partial v / \partial x-$ $\partial u / \partial y$ and $\partial h_{y} / \partial x-\partial h_{x} / \partial y$ which tend to turn them along the upper Alfvén direction. In the case of the velocity field, this turning occurs at the very small radius, $\nu / W_{+}$, whereas the turning of the magnetic field does not occur until the much larger radius $\kappa / W_{+}$.

At the smaller radius, a very peculiar thing happens. After the velocity field has begun to turn into the upper Alfven direction, there has been no equivalent action by the magnetic field to balance out the tendency of the velocity field to continue turning. Instead the combined interactions of velocity and magnetic fields now turn the velocity jet into the $\mathbf{U}$ direction, concentrating it and entraining an additional amount of fluid, $H_{x} / U$. Furthermore, this turning and entrainment induces in the surrounding flow a vortex of strength $H_{y} / U$ and an inward flow of strength $-H_{x} / U$. The magnetic field continues to be a simple pole.

As we go outward to radii of order $\kappa / W_{+}$, we find that the magnetic field turns and tends to point along the upper Alfvén direction. The jet now shifts its course so that it, too, points generally along the upper Alfvén direction. In so doing, it loses its entrained flow, returning to its original strength of unity. This turning and entrainment serves to cancel the outer source flow of strength $-H_{x} / U$ and the outer vortex flow of strength $H_{y} / U$. At very great distances compared to $\mathrm{k} / W_{+}$, the velocity and magnetic fields approach the pattern of Fig. 3 .

If we let $\nu$ go to zero, we see that the inner flow shrinks to zero and the picture becomes identical with that we had before for $\nu=0$. We now see that the apparent discrepancy between Tables III
TABLE VII. Strengths of the singularities for the various modes when $k=0$

\begin{tabular}{clccc}
\hline \hline Mode & & $\lambda$ & $\mu$ and $-\beta$ & $\chi$ \\
\hline & Source & 1 & 0 & 1 \\
$\begin{array}{c}\text { Strengths } \\
\text { of } \\
\text { singularities } \\
\text { for } \\
\text { unit } \\
\text { fundamental } \\
\text { solution }\end{array}$ & Portex & 0 & 1 & 0 \\
& Pole & $H_{x} / U$ & $H_{y} / U$ & $H_{x} / U$ \\
& $\begin{array}{l}\text { Irresistive } \\
\text { magnetic } \\
\text { wake } \\
\text { along U }\end{array}$ & $-\frac{U+H_{x}}{U}$ & $-\frac{H_{y}}{U}$ & $\frac{U-H_{x}}{U}$ \\
\hline
\end{tabular}

and VI is resolved. The inviscid slipstream of Table VI, which is not in Table III, conceals within itself a vortex and source flow that represents the difference between the other values given in the tables.

We could go on to discuss the behavior of the other modes, but since such a discussion would proceed along very similar lines to that just given for the $\lambda$ mode, we shall leave this as an exercise for the interested reader. For the same reason, we do not dwell at length upon the cases for which $\kappa$ is very much smaller than $\nu$, but content ourselves by giving in Table VII the strengths of the singularities when $\kappa=0$.

We are now in a position to summarize what we have found. When $\nu$ and $\kappa$ are equal, the four fundamental modes of the viscous-resistive operator are sources, vortices, poles, and currents which are distorted in such a way that they may be thought of as modified Alfvén waves that diffuse separately and independently as they travel outward. When $\nu$ becomes different from $\kappa$, the fundamental modes continue to be distorted sources, vortices, poles and currents which may still be thought of as diffusing waves. But now the primary wave, in addition to its normal diffusion [proportional to $2 \nu \kappa /(\nu+\kappa)$ ], experiences a differential diffusion (proportional to $\nu-\kappa)$. In the case of the $\lambda$ and $\chi$ modes, the primary waves are sources and poles whose field lines point jetlike along one or the other of the Alfven directions. When $\kappa>\nu$, the differential diffusion deflects the velocity jet toward the $U$ direction and induces secondary vortex and source flows. These secondary flows show strong distortions along the conjugate Alfvén directions. From a wave point of view, these secondary flows may be thought of as being generated by differential diffusion of the primary wave which excites diffuse secondary waves of the conjugate family (this explains why the secondary 
flows show strong distortions along the conjugate wave direction). These waves in turn excite tertiary waves, etc. The flow patterns of these secondary flows form a veil-like wake in the fan-shaped region between the two wave directions extending out from the singularity itself. This wake is fed from the primary wave which in turn is fed by the singularity at the origin.

If we go to very small values of $\nu$ compared to $\kappa$, the differential diffusion is so great that the original jet has been turned sufficiently that it emerges as a narrow slipstream in the $U$ direction. This subsequently turns and flows along one or the other of the Alfven directions. The fan-shaped wake now becomes quite prominent particularly for radii of order $\kappa / W_{+}$. However, it becomes diffuse and disappears for radii that are large compared to this. The magnetic field does not exhibit the complex behavior that the velocity field does, but when $\nu>\kappa$, the roles are reversed and the magnetic field becomes the principal actor.

Turning now to the $\mu$ and $\beta$ modes, the primary waves are vortices and currents which are distorted along one or the other of the Alfvén directions. Differential diffusion causes changes in these fields that are quite analogous to those for the $\lambda$ and $\chi$ fields. Consequently these modes, too, exhibit the new phenomenon of a fan-shaped wake downstream from the singularity. When $\nu \rightarrow 0$ or when $\kappa \rightarrow 0$, the $\mu$ and $\beta$ modes become identical and when both $\nu$ and $\kappa \rightarrow 0$, these modes disappear.

Before we conclude this section, it is worthwhile to say a few words about the implications of the above results for the more general theory of partial differential equations. Suppose we have an operator which can be factored into two independent operators. The modes of each of these independent operators will themselves be independent. But now if we alter the original operator (e.g., by varying some of the constants in it) so that it can no longer be factored, the modes will become intercoupled and their behavior patterns will become complicated combinations of the behavior patterns of the independent modes. Each will partake of some of the characteristics previously associated solely with the other. The significance of unfactorizability is thus that it represents an inextricable fusion of simple behavior patterns into more complex behavior patterns.

\section{FORCE LAWS FOR THE FUNDAMENTAL MODES}

An important question which we must now answer is: What are the forces acting upon these sources and vortices, poles and currents which characterize our field modes? An answer to this question will permit us to calculate the forces acting upon any object placed in the field if we know the pattern of sources and vortices, poles and currents which represent the body in the field.

The total stress tensor acting at any point within the medium is (see above)

$$
\mathbf{T}=\rho(-\mathrm{VV}-\mathrm{PI}+\nu \text { def } \mathbf{V})+\mathbf{B H}+\mathbf{E D} .
$$

If we integrate this over a surface surrounding each of our modal singularities, we obtain the force acting upon each of the singularities. When this is done, a simple result emerges. As would be expected, the conventional sources and vortices, poles and currents of Figs. 1 and 2 (i.e., those of the $\phi$ and $\psi$ modes) obey the force laws: If $Q$ is the strength of a source, $\Gamma$ is the strength of a vortex (counterclockwise field, $\Pi$ is the strength of a pole, and $J$ is the strength of a current (counterclockwise field), then the force on a source is $-\rho U Q$ along $\mathrm{J}$, the force on a vortex is $\rho U \Gamma$ along the right hand perpendicular to $\mathrm{U}$, the force on a pole is $H \Pi$ along $\mathbf{H}$, and the force on a current is $-H J$ along the right hand perpendicular to $\mathrm{H}$.

The sources and vortices, poles and currents which correspond to the viscous-resistive modes $(\lambda, \mu, \chi, \beta)$ obey the even simpler force laws: The force on a source is $-2 \rho U Q$ along $\mathbf{U}$, the force on a vortex is 0 , the force on a pole is $2 H$ II along $\mathbf{H}$, the force on a current is 0 .

This second set of force laws is unusual, and to the author's knowledge, has never been presented before. They may be summarized by saying that viscous-resistive sources and poles are twice as effective as conventional sources and poles in producing forces and they act in the same direction as their conventional counterparts. On the other hand, viscous-resistive vortices and currents are incapable of exerting forces of any kind.

The above results are surprising in that they show that when viscosity and resistivity are present, the ordinary force laws are not universally valid. In particular, it is usually accepted that the laws, $\mathbf{F}=\mathrm{J} \times \mathbf{H}$ and $\mathbf{F}=\Pi \mathbf{H}$, are cornerstones in the theory of the magnetic field. These are valid for the $\phi$ and $\psi$ modes, but not for the $\lambda, \mu, \chi$, and $\beta$ modes.

The results of Hasimoto ${ }^{2}$ for the magnetohydrodynamic extension of the Kutta-Joukowski-Filon integral theorem for the forces on an object may be obtained from the above force laws by assuming that there are no resultant sources or magnetic 
poles in the flow, i.e., that the total strengths of the conventional sources and poles just balance those of the viscous-resistive sources and poles.

\section{BOUNDARY LAYERS AND WAKES IN MAGNETOHYDRODYNAMICS}

The concept of boundary layers and wakes is of such great importance in fluid mechanics that it is incumbent upon us to ask what happens to these concepts in magnetohydrodynamics. First we must show how the concept of fundamental modes treats the boundary layer and wake problem in ordinary fluid mechanics. Then we shall apply the same techniques to the magnetohydrodynamic field.

For the fluid mechanics field equivalent to our magnetohydrodynamic field, we have the equations

$$
\begin{array}{r}
U \frac{\partial u}{\partial x}-\nu \nabla^{2} u+\frac{\partial P}{\partial x}=0 \\
U \frac{\partial v}{\partial x}-\nu \nabla^{2} v+\frac{\partial P}{\partial y}=0 \\
\frac{\partial u}{\partial x}+\frac{\partial v}{\partial y}=0 .
\end{array}
$$

These equations have four modes of behavior. If we introduce the four modal variables $\phi, \psi, \lambda, \mu$, which satisfy the equations $\nabla^{2} \phi=0, \nabla^{2} \psi=0$, $U(\partial \lambda / \partial x)-\nu \nabla^{2} \lambda=0$ and $U(\partial \mu / \partial x)-\nu \nabla^{2} \mu$, then the modes may be expressed in the form given in Table VIII. The fundamental solutions for $\phi$ and $\psi$ are $(1 / 2 \pi) \log r$ and for $\lambda$ and $\mu$ are $[\exp (R \cos \theta)] K_{0}(R)$, where $R=U r / 2 \nu$. The $\phi$ solution gives a conventional source as shown in Fig. 1 and the $\psi$ solution gives the conventional vortex shown in Fig. 2. The $\lambda$ solution gives a viscous source of the type shown in Fig. 3 except that here the axis of the singularity points along $U$. Similarly, the $\mu$ solution is the viscous vortex of Fig. 4 with its axis pointing along $\mathbf{U}$.

Neither the $\phi$ nor $\psi$ modes lead to boundary layer or wake phenomena. To see how such concepts arise from the $\lambda$ and $\mu$ modes, consider the traditional

Table VIII. Modes of the viscous flow field.

\begin{tabular}{ccccc}
\hline \hline & $\phi$ & $\psi$ & $\lambda$ & $\mu$ \\
\hline$v$ & $\frac{\partial \phi}{\partial x}$ & $\frac{\partial \psi}{\partial y}$ & $U \lambda-\nu \frac{\partial \lambda}{\partial x}$ & $\nu \frac{\partial \mu}{\partial y}$ \\
$P$ & $\frac{\partial \phi}{\partial y}$ & $-\frac{\partial \psi}{\partial x}$ & $-\nu \frac{\partial \lambda}{\partial y}$ & $-\nu \frac{\partial \mu}{\partial x}$ \\
\hline
\end{tabular}

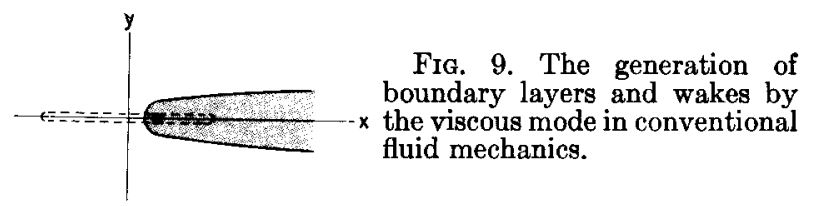

thin plate of boundary layer theory as shown in Fig. 9. Here the plate is shown dotted and we have singled out a small section of the plate in order to examine its influence upon the flow field. If the nature of the plate is such that it calls into play either the $\lambda$ or the $\mu$ modes (and generally this will be necessary to meet the boundary conditions at the plate) the resulting flow field from these modes will be confined to the shaded area shown. Clearly, the $\lambda$ and $\mu$ disturbances are carried back over the body by the main stream and trail off downstream. If the Reynolds number of the body, $U L / \nu$, is small, the field from all of the $\lambda$ and $\mu$ disturbances along the body will be confined to thin layers flowing over the body. Furthermore, their flow field out behind the body will also be thin. These thin layers at high Reynolds numbers are the boundary layers and wakes of ordinary fluid mechanics.

Let us turn to the magnetohydrodynamic field. We have seen earlier that the $\lambda$ and $\mu$ modes of ordinary fluid mechanics undergo a metamorphosis and become the $\lambda, \mu, \chi$, and $\beta$ modes of magnetohydrodynamics. Any boundary layer or wake phenomena will appear in these modes and not in the $\phi$ or $\psi$ modes.

In Sec. VIII we explored these modes quite carefully for the case $\nu=\kappa$. We found that for this case no boundary layer or wake phenomena can possibly exist. Viscous-resistive phenomena are confined to the Alfvén waves which diffuse independently out in the flow field. There is no evidence of any viscous-resistive flow field which washes back over a body to form a boundary layer or a wake.

Next we must ask if boundary layers or wakes are possible when $\nu$ is not equal to $\kappa$. In Sec. IX, where we explored this case, we found a result that indicates that the strict answer to this question is no. There we found that for all values of $\nu$ and $k$, the viscous resistive field patterns at great distances from the disturbances are the same as those for the case $\nu=\kappa$. Since a great distance is one for which the viscous and resistive Reynolds numbers are large, we can never have boundary layers and wakes in the conventional sense.

However, an interesting case is possible. In Sec. IX it is seen, for example, that when $\kappa$ is much greater 
than $\nu$, the fluid emerging from the viscous-resistive singularities flows in a thin jet or wake in the $\mathrm{U}$ direction. This flow persists until the Reynolds number $W r / \kappa$ reaches a modest value (finite but small compared to unity). Thus for the case in which the Reynolds number $W L / \nu$ is large, but the Reynolds number $W L / \kappa$ is small, velocity boundary layers on the plate will exist. If the conditions on $\nu$ and $\kappa$ are reversed, then magnetic boundary layers on the plate will exist.

Why is it that conventional boundary layers and wakes no longer exist in magnetohydrodynamics? As Alfvén has shown, ${ }^{1}$ the magnetic field acts to prevent a simple shear from taking place across the magnetic lines. It thus prevents the main velocity field from carrying the shear disturbances of the viscous-resistive modes back over the body to form boundary layers and wakes. Instead, such disturbances are carried off as Alfvén waves. Thus, diffuse Alfvén waves inherit the rights and privileges formerly exercised by boundary layers and wakes. When the resistivity is large, the scale of magnetic diffusion can be so great that a special type of velocity boundary layer is possible. However, it diffuses and disappears completely at distances for which the magnetic Reynolds number becomes large.

\section{CONCLUSION}

The method of field modes has proven to be a powerful tool in exploring the difficult and challenging problems of magnetohydrodynamics. There is reason to hope that it can be used in exploring other fields with equal success.

\section{ACKNOWLEDGMENT}

This research was supported by the U. S. Air Force through the Air Force Office of Scientific Research of the Air Research and Development Command, under Contract number AF 49(638)-496. 